6-1-1998

\title{
Translational Diffusion of Small and Large Mesoscopic Probes in Hydroxypropylcellulose-Water in the Solutionlike Regime
}

\author{
Kiril A. Streletzky \\ Cleveland State University, K.STRELETZKY@csuohio.edu
}

George D.J. Phillies

Worcester Polytechnic Institute, phillies@wpi.edu

Follow this and additional works at: https://engagedscholarship.csuohio.edu/sciphysics_facpub

Part of the Physics Commons

How does access to this work benefit you? Let us know!

Publisher's Statement

(C) 1998 American Institute of Physics

\section{Repository Citation}

Streletzky, Kiril A. and Phillies, George D.J., "Translational Diffusion of Small and Large Mesoscopic Probes in Hydroxypropylcellulose-Water in the Solutionlike Regime" (1998). Physics Faculty Publications. 252.

https://engagedscholarship.csuohio.edu/sciphysics_facpub/252 


\title{
Translational diffusion of small and large mesoscopic probes in hydroxypropylcellulose-water in the solutionlike regime
}

\author{
Kiril A. Streletzky and George D. J. Phillies ${ }^{\text {a) }}$ \\ Department of Physics, Worcester Polytechnic Institute, Worcester, Massachusetts 01609
}

(Received 24 April 1997; accepted 10 November 1997)

\begin{abstract}
Quasi-elastic light scattering spectroscopy was used to study the translational diffusion of monodisperse spheres in aqueous $1 \mathrm{MDa}$ hydroxypropylcellulose (HPC) at $25^{\circ} \mathrm{C}$. Probe diameters $d$ spanned 14-455 nm; HPC concentrations were $0 \leqslant c \leqslant 7 \mathrm{~g} / \mathrm{L}$. Light scattering spectroscopy consistently found spectra having the form $g^{(1)}(t)=\left(1-A_{f}\right) \exp \left(-\theta t^{\beta}\right)+A_{f} \exp \left(-\theta_{f} t^{\beta_{f}}\right)$. Here $\theta_{f}$ and $\beta_{f}$ refer to the "fast" mode; $\theta$ and $\beta$ describe the "slow" mode. We examine the dependence of $\theta, \beta, \theta_{f}, \beta_{f}$, and $A_{f}$ on $d, c$, scattering vector $q$, and viscosity $\eta . \beta=1$ for large probes; elsewise, $\beta$ and $\beta_{f}$ are $\in(0,1)$. The slow mode, with short-lived memory function, is diffusive; for large probes $\theta \approx(d \eta)^{-1}$. The fast mode, with long-lived memory function, appears coupled to polymer chain internal dynamics. Probe behavior differs between "small" and "large" probes. Small probes have diameters $d<R_{h}, R_{h}$ being the chain hydrodynamic radius. Large probes have $d \geqslant R_{g}, R_{g}$ being the polymer radius of gyration. (C) 1998 American Institute of Physics. [S0021-9606(98)50507-7]
\end{abstract}

\section{INTRODUCTION}

Polymer dynamics in non-dilute solutions remains an important problem of macromolecular science. Several models treat polymer transport. A widely-accepted model is the reptation/scaling picture of de Gennes ${ }^{1}$ and Doi and Edwards. ${ }^{2}$ Reptation predictions are based on entanglement (topological) forces and assume power law dependences of the polymer self-diffusion coefficient $D_{s}$ and polymer viscosity $\eta$ upon polymer concentration $c$ and molecular weight $M$.

Numerous literature reviews ${ }^{3-5}$ identify inconsistencies between experiment and reptation/scaling predictions for $D_{s}$ and $\eta$ in the semidilute region $c>c^{*}$. Reptation predicts $D_{s} \sim M^{-2} c^{-2}$ and $\eta \sim M^{3} c^{4}$ for $c>c^{*}$, while empirically ${ }^{3,5}$

$$
D_{s}=D_{0} \exp \left(-a c^{\nu} M^{\gamma}\right)
$$

and

$$
\eta=\eta_{0} \exp \left(a c^{\nu^{\prime}} M^{\gamma^{\prime}}\right) .
$$

Here $a$ is a scaling pre-factor, and $\nu, \nu^{\prime}, \gamma$, and $\gamma^{\prime}$ are scaling exponents. Phillies demonstrated ${ }^{3}$ that equations 1 and 2 describe the literature very well for many polymer solutions over a wide range of concentrations. Although the reptation prediction for self-diffusion $D_{s} \sim M^{-2}$ appears to be confirmed experimentally for polymer melts, the wellestablished experimental behavior $\eta \sim M^{3.4}$ differs substantially from the originally-predicted behavior. ${ }^{4}$

There are several alternatives to the reptation/scaling model of polymer dynamics; see Refs. 3,5. The hydrodynamic scaling model of Phillies ${ }^{3,5}$ is a successful alternative; this model assumes that interchain hydrodynamic interactions dominate entanglements and derives Eq. 1. An impor-

\footnotetext{
a) Author to whom correspondence should be addressed; Electronic mail: phillies@wpi.wpi.edu (Internet).
}

tant implication of the hydrodynamic scaling model is a solutionlike-meltlike transition with increasing $c$. At this transition, the phenomenological concentration and molecular-weight dependencies of $D_{s}$ and $\eta$ change from a stretched-exponential behavior (in $c, M$ ) in more dilute solution to a power-law (in $M$ ) behavior in more concentrated solution. The transition concentration $c^{+}$is the concentration above which Eq. 2 is replaced by

$$
\eta=\bar{\eta} c^{x}
$$

A review ${ }^{5}$ and experimental studies by Phillies et al. ${ }^{6,7}$ find this change in $\eta$ from solutionlike (stretched-exponential) behavior to meltlike (power-law) behavior in many though not all systems. ${ }^{5,6}$ In most cases considered in ref. 5, $D_{s}$ follows Eq. 1 for all concentrations, including $c>c^{*}$.

The coupling model of Ngai and collaborators ${ }^{8,9}$ considers the process of relaxation in complex systems as the "cooperative process of motions coupled together by interactions." 9 This model also predicts that $D_{s}$ follows Eq. 1. In this model, dynamic constraints (the degree of coupling) between polymer chains play an important role in relaxation. In dilute solutions, there is almost no coupling; the degree of coupling increases with increasing concentration. $^{8-11}$

There is substantial experimental support for the hydrodynamic scaling model (refs. 3,5-7,12,13, and 14 and references therein). Experimental support for the Ngai coupling model as applied to polymers has also been reported..$^{8-11,15,16}$

This paper treats the diffusion of spherical polystyrene latex particles (PSL), used as optical probes, in solutions of the uncharged, semirigid, water-soluble polymer hydroxypropylcellulose (HPC) of high molecular weight. The probes had a wide range of diameters $(14 \mathrm{~nm} \leqslant d \leqslant 455 \mathrm{~nm})$. Quasi-elastic light scattering spectroscopy (QELSS) was used to observe the motion of the dilute, strongly-scattering 
probes in solutions of the weakly-scattering polymer. Previously, ${ }^{7,13}$ we inferred the self-diffusion coefficient $D_{p}$ of probes from the initial slope of the QELSS spectrum $S(q, t)$. However, if $S(q, t)$ is substantially non-exponential, the initial slope does not describe the spectrum completely. In this paper we extend our previous studies by using direct line shape analysis to characterize probe spectra.

Probe diffusion measurements in HPC solutions have also been reported by Brown and Rymden, ${ }^{17}$ Yang and Jamieson, ${ }^{18}$, Russo et al.,${ }^{19-21}$, Bu and Russo, ${ }^{12}$ and Phillies and Lacroix. ${ }^{22} \mathrm{Bu}$ and Russo ${ }^{12}$ studied diffusion of 10 different size probes in HPC of high molecular weight; their work used smaller probes $\left(0.5 \mathrm{~nm} \leqslant R_{h} \leqslant 55.1 \mathrm{~nm}\right.$, where $R_{h}$ is the probe hydrodynamic radius) than we did. Studies by Phillies et al. ${ }^{7,13}$ examined high molecular-weight HPC, but used only one probe size. Reference 22 used four probes having diameters similar to those studied here, but it studied only intermediate $(300 \mathrm{kDa})$ molecular-weight $\mathrm{HPC}$, not the 1 MDa HPC studied here.

Viscosity measurements on HPC: water are given in Refs. 7,12,18,21. A detailed viscosity study is reported by Phillies and Quinlan. ${ }^{6}$ For 1 MDa HPC solution, Phillies and Quinlan ${ }^{6}$ found that $\eta(c)$ has a very sharp solutionlikemeltlike transition at $c^{+}=6 \mathrm{~g} / \mathrm{L}$ and $\eta \approx 144 \mathrm{cP}$. They also explicitly show that $\eta(c)$ is continuous and analytic through the transition, i.e., $\eta(c)$ and $\partial \log (\eta(c)) / \partial \log (c)$ are both continuous at $c^{+}$. At $c<c^{+}, \eta$ followed a stretched exponential $\eta_{0} \exp \left(\alpha c^{\nu}\right)$ with $\eta_{0}=0.85, \alpha=0.97$ and $\nu=0.93$; at $c>c^{+}, \eta$ followed a power law, Eq. 3, with $x=4.33$.

This paper addresses: (1) Determination of the detailed form of the spectral line shape, which is found to have a bimodal relaxation. The angular and concentration dependencies of both modes are carefully examined. (2) Measurement and analysis of spectral line shape parameters of probes diffusing in HPC: water, in the solutionlike regime and near the solutionlike-meltlike transition. (3) Determination of the effect of probe size on spectral line shape.

The next section describes our apparatus. Further sections discuss line shape analysis, describe results and data interpretation, and discuss our findings with respect to the literature. A discussion with conclusions closes the paper.

\section{EXPERIMENTAL METHODS}

The subject of this study was hydroxypropylcellulose (HPC), nominal molecular weight $1 \mathrm{MDa}$, from Scientific Polymer Products. Stock solutions of polymer concentration $7 \mathrm{~g} / \mathrm{L}$ were initially prepared in water purified (resistivity $14-18 \mathrm{M} \Omega / \mathrm{cm}$ ) by Millipore Milli-RO and Milli-Q water systems. Other solutions were prepared by serial dilution to cover polymer concentrations of $0-7 \mathrm{~g} / \mathrm{L}$. A trace surfactant concentration (0.2 wt. \% TX-100 (Aldrich)) was added to prevent HPC absorption by probes. The TX-100 concentration was chosen based on Phillies et al. ${ }^{5}$

Nominal diameters of the probes were 14, 21, 38, 87, 189, and $282 \mathrm{~nm}$ (Interfacial Dynamics), $67 \mathrm{~nm}$ (Seradyn), and $455 \mathrm{~nm}$ (Dow Chemicals). Trace quantities (5-10 $\mu \mathrm{L}$ of carboxylate-modified polystyrene latex spheres (PSL) per $\mathrm{mL}$ of polymer solution) were used in the probe diffusion experiments. Since latex spheres are very good scatterers, even trace amounts of PSL are usually enough for probes to dominate the scattering intensity. Multiple scattering by the probes was avoided by using probe volume fractions under 0.001 .

To interpret spectra, it is essential that the observed relaxations correspond to probe motion, not to concentration fluctuations of the polymer or surfactant. As control experiments, we compared under identical operating conditions spectra of probes in polymer solutions and spectra of probefree polymer solutions. Spectra of probe-free polymer solutions are far weaker than spectra of polymer solutions containing probes. For probes with $d \geqslant 50 \mathrm{~nm}$, matrix scattering at all $t$ is less than $1 \%$ of probe scattering. For smaller $(d<50 \mathrm{~nm})$ probes at high HPC concentration, polymer scattering is more substantial, but probes scatter much more light than polymer does. For most of the probes and HPC concentrations, the initial amplitude of the spectrum of a probe-free polymer solution is $\leqslant 1 \%$ of the spectrum of a probe:polymer solution. For the smallest spheres $(14 \mathrm{~nm})$ at the highest HPC concentration $(7 \mathrm{~g} / \mathrm{L})$ the initial spectral amplitude of the polymer solution is less than $4 \%$ of the probe:polymer spectral amplitude. The amplitude ratio depends on the time scale. The spectrum of a probe:polymer sample decays somewhat faster than the spectrum of a probe-free polymer sample. To confirm that polymer scattering does not influence our spectral analysis we measured probe:polymer and probe-free polymer spectra under the same conditions (for the smallest $14 \mathrm{~nm}$ spheres), subtracted (at the field correlation level) the polymer spectrum from the probe:polymer spectrum, and used our fitting procedure to analyze the difference spectra. We found for all HPC concentrations, at the time scales covered by this study, that the difference spectrum is successfully fit by exactly the same function and parameters that we obtained by fitting the probe-polymer mixtures without subtraction. The difference in fitting parameters before (probe:polymer spectrum) and after subtraction (probe-free polymer spectrum subtracted from probe: polymer spectrum) was on the order of the experimental error. Therefore polymer scattering does not influence our probe spectra significantly.

For the smallest $14 \mathrm{~nm}$ spheres, in addition to comparing probe-free polymer solutions with solution containing probes at our standard probe concentration $x$, we conducted additional control experiments using triple probe concentration $3 x$. At all $t$, the amplitude of the spectra of the probeconcentration $3 x$ samples was roughly 3 times higher than the amplitude of the probe-concentration $x$ samples, confirming that we are monitoring probe motions. The (measurable) polymer spectra, while not negligible on all time scales, do not perturb our determinations of the spectral line shape for probe systems. Our spectra thus reflect to high accuracy probe motions in the polymer matrix.

Probe: polymer: surfactant solution samples were clarified by passage through cellulose filters (Micron Separations, pore diameters $0.22-2.0 \mu \mathrm{m}$ ). The pore size was selected using two criteria. First, the pore size must be larger than the probe diameter so the probes pass through the filter. Second, HPC solutions of high concentration $(5-7 \mathrm{~g} / \mathrm{L})$ are very viscous. To limit filtration time to 30-45 minutes, we used 
filters with pores $2-3$ times larger than the probe size. Light scattering cells were glass fluorimeter cuvettes (NSG Precision Cells, Inc), four sides polished. Cells were thoroughly rinsed several times with $18 \mathrm{M} \Omega$ conductivity grade water passed through an $0.22 \mu \mathrm{m}$ filter; cells were dried by nitrogen clarified with an $0.2 \mu \mathrm{m}$ filter.

Quasi-elastic light scattering spectroscopy (QELSS) studies the fluctuating light scattering intensity $I(q, t)$ by determining the intensity-intensity correlation function $S(q, \tau)$. Here $q$ is the magnitude of the scattering vector,

$$
q=\frac{4 \pi n}{\lambda} \sin \frac{\theta}{2},
$$

where $n$ is the index of refraction, $\lambda$ is the laser light wavelength in vacuo, and $\theta$ is the scattering angle.

The intensity-intensity correlation $S(q, \tau)$ function for a light scattering experiment with duration $T$ is

$$
S(q, \tau)=\int_{0}^{T} d t I(q, t) I(q, t+\tau),
$$

where $\tau$ is a shift in time. For a linear correlator, $\tau$ can be represented as series of $k$ adjoining time intervals having equal durations $\delta \tau$ (sample time). Operationally, a digital correlator counts the number of received photons $n_{j}$ during each interval of width $\delta \tau$, and computes the intensityintensity correlation function as a summation over $K=T / \delta \tau$ time intervals

$$
S(q, \tau)=\sum_{j=1}^{K} n_{j} n_{j+k} .
$$

In our experiments, spectra were analyzed by a $264-$ channel Brookhaven Instruments BI2030AT digital multitau correlator. The multiple sample time (multitau) option of the correlator was effectively used in our measurements to monitor simultaneously fast $\left(1-10^{2} \mu \mathrm{sec}\right)$ and slow $\left(10^{4}-10^{6} \mu \mathrm{sec}\right)$ relaxation processes. We confirmed that the measured spectrum had decayed down to the baseline, as inferred from the correlator delay channels. The multitau option allows one to split the 264 real-time data channels into four groups, each with a different sample time of value $2^{l} \delta \tau$ (where $l=0$ in the first bank of channels and $l$ is integer $l_{i} \geqslant l_{i-1} \geqslant 0$ for $\left.i=2 \ldots 4\right)$. Details of interpreting the nominal $\tau$, as calculated by a multitau correlator, were discussed by Ref. 23. For each sample we made at least three measurements under identical experimental conditions to ensure reliability of the results. Overall, we measured more than 1000 light scattering spectra.

Most measurements used a Spectra-Physics 2020-03 $\mathrm{Ar}^{+}$laser with maximum power output of $1.5 \mathrm{~W}$ at 514.5 $\mathrm{nm}$, coupled to a BI-200SM photometer-goniometer (Brookhaven Instruments). Cells were placed into a decalin-filled index-matching vat. A Neslab RTE-110 temperature regulator maintained samples at $25 \pm 0.1^{\circ} \mathrm{C}$. Most of the experiments were conducted at scattering angle $\theta=90^{\circ}$ $\left(q=2.30 \times 10^{5} \mathrm{~cm}^{-1}\right)$. We also studied the $q$-dependence of $S(q, \tau)$. Rotation of the detector arm covered internal scattering angles $28^{\circ} \leqslant \theta \leqslant 107^{\circ}$, i.e. $7.84 \times 10^{4} \leqslant q \leqslant 2.61 \times 10^{5}$ $\mathrm{cm}^{-1}$. For latex sphere probes of diameter 21, 87, and 189 $\mathrm{nm}$ and solutions with concentrations $0-5 \mathrm{~g} / \mathrm{L}(0-6 \mathrm{~g} / \mathrm{L}$ for $87 \mathrm{~nm}$ spheres) we made angular experiments starting with external arm angle $\theta_{e}=105^{\circ}$, and decrementing $\theta_{e}$ by $5^{\circ}$ down to $30^{\circ}$ ( $45^{\circ}$ was inaccessible).

In some angular-dependence experiments, we illuminated sample cells with a $50 \mathrm{~mW}$ Coherent Radiation DPSS532 diode-pumped frequency-doubled $\mathrm{cw}$ laser operating at $532 \mathrm{~nm}$. In these studies, the detector was an RCA 7265 photomultiplier tube, mounted on an arm on a Model 496 Power Rotation Stage (Newport), controlled by Model 855 Programmable Controller System (Newport). External scattering angles ranged from 10 to $90^{\circ}$ at $5^{\circ}$ intervals except for 40, 45, and $50^{\circ}$. A computer-controlled Neslab RTE-100 bath connected to a massive copper cell-holder gave a sample temperature $25 \pm 0.1^{\circ} \mathrm{C}$.

\section{DATA ANALYSIS}

We analyzed spectra by fitting them to specific functional forms, as opposed to fitting to a generic form such as Koppel's cumulants expansion. ${ }^{24}$ This section explains how and why we chose our forms. First, the relationship between the measured spectrum $S(q, t)$ and the field correlation function $g^{(1)}(q, t)$ is noted. The numerical process for parameter optimization within $g^{(1)}(q, t)$ is considered. Second, a review of the literature on probe diffusion in HPC: water confirms that our approach is consistent with other work. Third, we discuss the forms that we tried. For small spheres a sum of two stretched exponentials fits spectra well. For large spheres, analysis of the $q$-dependence of $g^{(1)}(q, t)$ confirmed that a sum of a faster stretched exponential and a slower simple exponential was the best available fitting function.

We first consider spectral analysis. The intensityintensity correlation function $g^{(2)}(q, t)$ is related to the fieldcorrelation function $g^{(1)}(q, t)$ via

$$
g^{(2)}(q, t)=S(q, t)-B=A\left(g^{(1)}(q, t)\right)^{2},
$$

where $A$ is the scattering amplitude and $B$ is the baseline, the time-independent part of the spectrum. We determined $B$ as an average of $S(q, t)$ in 6 correlator channels located near $t=1024 \times 2^{l} \delta t$, where $l=l_{4}$. We tried different forms of $g^{(1)}(q, t)$ to see which one best described $g^{(2)}(q, t)$. We minimized $\left[g^{(2)}(q, t)-S(q, t)\right]^{2} /[S(q, t)]^{2}$ using nonlinear least squares and the simplex algorithm. ${ }^{25}$ During the fitting process, we tried multiple initial values for parameters to determine if the output of the simplex algorithm depended upon the initial values. If the final fit parameters changed insignificantly (less than 1-2\%) when the initial parameter values were changed, we considered the fit to be stable; we elsewise characterize the fit as "unstable."

Spectral line shapes for probe diffusion in HPC: water have been determined previously. Phillies et al. ${ }^{7}$ studied spherical probes in solutions of HPC with nominal molar masses of $60,100,300$ and $1000 \mathrm{kDa}$. Phillies et al. ${ }^{7}$ successfully fitted $S(q, t)$ using a stretched exponential fieldcorrelation function

$$
g^{(1)}(q, t)=\exp \left(-\theta t^{\beta}\right) .
$$

Here $\theta$ is the decay pseudorate and $\beta$ is the stretching exponent. Reference 7 found that fits of $g^{(1)}(q, t)$ to Eq. 8 were 
very good for 60 and $100 \mathrm{kDa}$ HPC solutions. Spectra were nearly pure-exponential $(0.85 \leqslant \beta \leqslant 1)$. Probes in $300 \mathrm{kDa}$ HPC showed good agreement with Eq. 8 with $0.65 \leqslant \beta \leqslant 1$. Finally, for $1 \mathrm{MDa}$ HPC, Phillies, et al. ${ }^{7}$ show $g^{(1)}(q, t)$ could be described by Eq. 8, but note a possible second slow decay mode.

Brown and Rymden, ${ }^{17}$ and Mustafa and Russo ${ }^{19}$ found a bimodal relaxation in spectra of probes in HPC. Brown and Rymden ${ }^{17}$ used multiexponential analysis of the spectral lineshape for probes in $800 \mathrm{kDa}$ HPC. Mustafa and Russo ${ }^{19}$ used multiexponential analysis and two different Laplace inversion methods for probes in $300 \mathrm{kDa}$ HPC. Reference 17 and all three methods in Ref. 19 revealed the same result: two relaxation modes were present in each spectrum.

Studying probe diffusion in $300 \mathrm{kDa}$ HPC, Phillies and Lacroix ${ }^{22}$ tried a form due to Nystrom et al. ${ }^{11}$

$$
g^{(1)}(q, t)=A_{f} \exp \left(-\theta_{f} t\right)+\left(1-A_{f}\right) \exp \left(-\theta t^{\beta}\right) .
$$

Here $A_{f}$ is the amplitude fraction of the fast mode and $\theta_{f}$ is the decay rate of the fast mode. Phillies and Lacroix ${ }^{22}$ discovered that Eq. 9 is significantly better than Eq. 8 for probes in $300 \mathrm{kDa}$ HPC at $c>10 \mathrm{~g} / \mathrm{L}$; the fast decay was weak $\left(0.01 \leqslant A_{f} \leqslant 0.03\right)$, so deviations from Eq. 8 were not clearly pronounced.

We concentrated on obtaining very accurate measurements of $S(q, t)$ and finding an optimal functional form for $g^{(1)}(q, t)$. Using the correlator multitau option, we covered 4-6 decades (all that were needed) in time and a 100 to 5000 fold decay of $g^{(2)}(q, t) / g^{(2)}(q, 0)$. We used a very small sample time for the first data channel bank: $1 \mu \mathrm{sec}$ for low HPC concentrations $(c \leqslant 3 \mathrm{~g} / \mathrm{L})$ and small spheres $(d \leqslant 67$ $\mathrm{nm}$ ), and 2-8 $\mu \mathrm{sec}$ in order to cover large delay times adequately for higher concentrations and larger spheres.

We find different diffusive behaviors for probes of different sizes. Small spheres (diameter $d<67 \mathrm{~nm}$ ) gave an obviously bimodal spectrum at all $c$. Spectra of large spheres $(d>67 \mathrm{~nm})$ do not have an obvious bimodal line shape, but are found to be bimodal in numerical analysis of each spectrum. Spheres of $67 \mathrm{~nm}$ exhibit some properties of each regime. Modestly different forms of $g^{(1)}(q, t)$ were adequate to describe the spectra of large and of small probes. Our rationale for identifying these regimes follows.

For small probe diameters $(14,21$, and $38 \mathrm{~nm})$, one sees a clear bimodal relaxation at every polymer concentration $c>0 \mathrm{~g} / \mathrm{L}$ studied. Figure 1 show a typical spectrum. Fits of such spectra to Eq. 8 fail badly; rms fractional errors were $1 \times 10^{-2}-4 \times 10^{-2}$. We also fit spectra for small spheres to Eq. 9. The non-exponentiality of the fast decay keeps Eq. 9 from fitting these spectra; rms fractional errors were $2 \times 10^{-3}-5.5 \times 10^{-3}$. Figure 1a shows an exemplary fit to Eq. 9. At $t \geqslant 10^{3} \mu \mathrm{sec}$, Eq. 9 does not fit this spectrum well. However, this spectrum is described well by a sum of two stretched exponentials, namely

$$
g^{(1)}(q, t)=A_{f} \exp \left(-\theta_{f} t^{\beta_{f}}\right)+\left(1-A_{f}\right) \exp \left(-\theta t^{\beta}\right) .
$$

Here $\beta_{f}$ and $\theta_{f}$ are a stretching exponent and a relaxation pseudorate of the fast decay. Figure $1 \mathrm{~b}$ shows Eq. 10 fit to

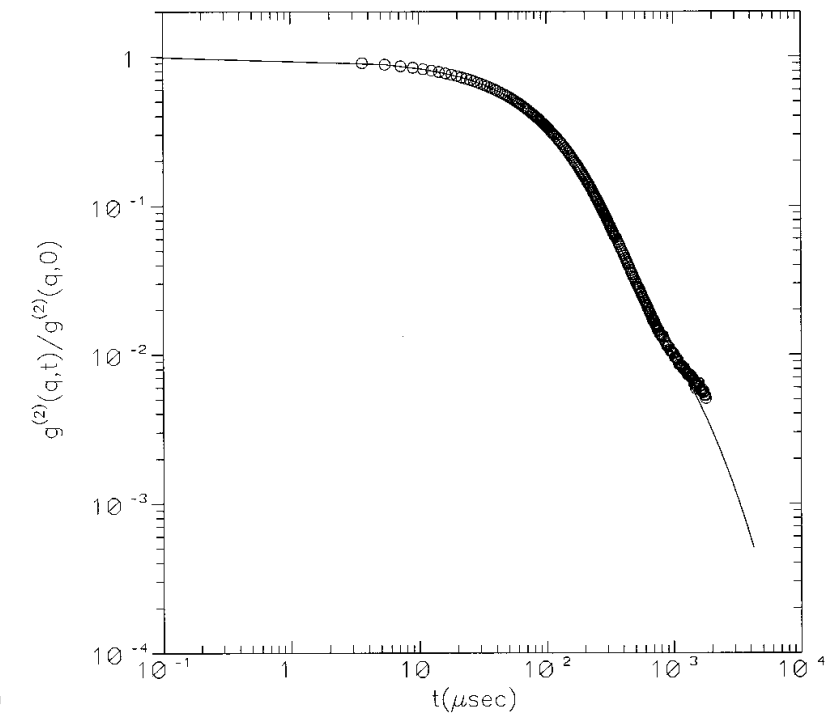

(a)

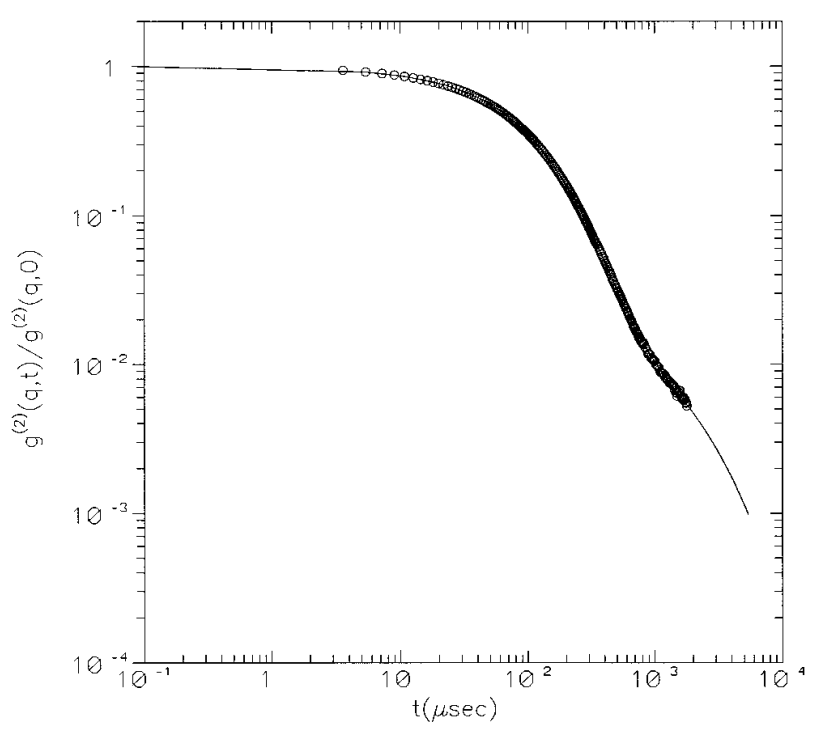

FIG. 1. Typical spectrum of $14 \mathrm{~nm}$ diameter polystyrene latex spheres in 3 g/L HPC: water. Solid line represents a fit to the spectrum using $g^{(1)}(q, t)$ in the form of: (a) a sum of a stretched exponential and a fast pure exponential (Eq. 9), (b) a sum of two stretched exponentials (Eq. 10). Fit parameters are in Table I.

$S(q, t)$ of $14 \mathrm{~nm}$ probes in $3 \mathrm{~g} / \mathrm{L}$ HPC. Eq. 10 (solid line) accurately describes $S(q, t)$. Fitting parameters from the above analysis appear in Table I.

For small spheres ( $d$ of 14,21 , and $38 \mathrm{~nm}$ ) at all concentrations, Eq. 10 fits each spectrum well over its entire decay. Rms fractional errors were in the range

TABLE I. Parameters from fits to a representive spectrum for small $(d=14$ $\mathrm{nm})$ PSL spheres in $3 \mathrm{~g} / \mathrm{L}$ HPC: water solution, using for $g^{(1)}(q, t)$ : (1) a single stretched exponential (Eq. 8), (2) a sum of a slow stretched exponential and a fast pure exponential (Eq. 9), and (3) a sum of two stretched exponentials (Eq. 10).

\begin{tabular}{ccccccc}
\hline \hline Form & \multicolumn{1}{c}{$\theta$} & \multicolumn{1}{c}{$\beta$} & \multicolumn{1}{c}{$A_{f}$} & $\theta_{f}$ & $\beta_{f}$ & rms error \\
\hline 1 & $1.55 \times 10^{-2}$ & 0.77 & 0 & $\mathrm{n} / \mathrm{a}$ & $\mathrm{n} / \mathrm{a}$ & $2.17 \times 10^{-2}$ \\
2 & $0.61 \times 10^{-2}$ & 1 & 0.41 & $4.48 \times 10^{-2}$ & 0.50 & $3.11 \times 10^{-3}$ \\
3 & $1.02 \times 10^{-2}$ & 0.89 & 0.19 & $1.32 \times 10^{-2}$ & 0.57 & $9.15 \times 10^{-4}$ \\
\hline \hline
\end{tabular}




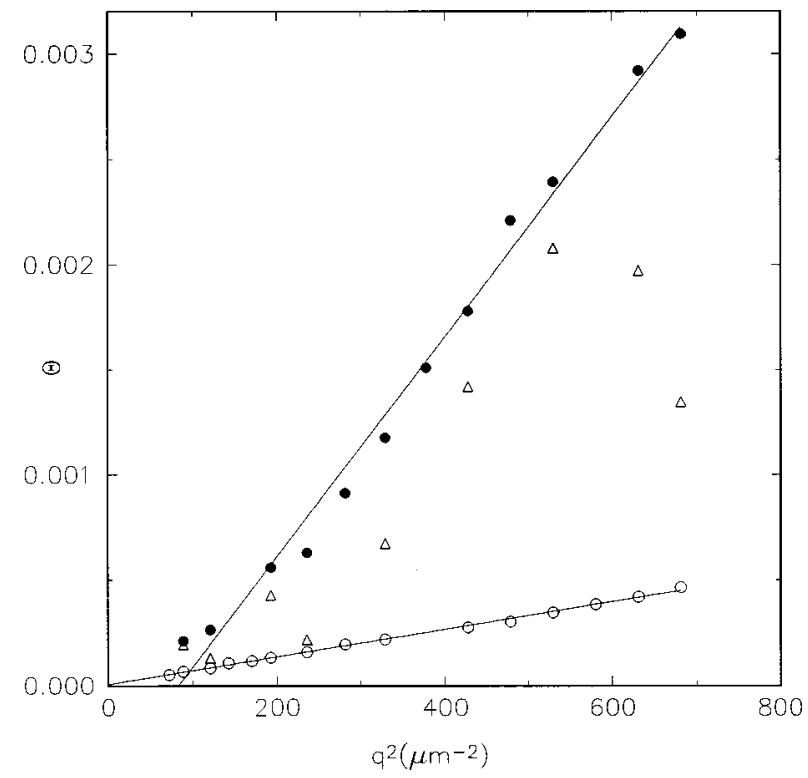

FIG. 2. The slow decay pseudorate $\theta$ as a function $q^{2}$, for $87 \mathrm{~nm}$ probes in $4 \mathrm{~g} / \mathrm{L}$ HPC. Filled circles are $\theta$ from Eq. 9; triangles are $\theta$ from Eq. 10; open circles are $\theta$ from Eq. 11, confirming our use of Eq. 11. Units of $\theta$ are $(\mu S)^{-\beta}$.

$5.5 \times 10^{-4}-1.3 \times 10^{-3}$. The slower relaxation dominates $g^{(1)}$ when $g^{(2)}(q, t) / g^{(2)}(q, 0) \leqslant 0.04$. At earlier times, the fast relaxation is dominant. Depending on $c$ and $d, \theta_{f} / \theta$ was in the range 1.3 to 11 ; the modes remain separable at small $\theta_{f} / \theta$ because the stretching parameters were very different $\left(0.7 \leqslant \beta \leqslant 1,0.2 \leqslant \beta_{f} \leqslant 0.6\right)$. The fraction $A_{f}$ of the fast decay at $90^{\circ}$ scattering was in the range $0.20-0.35$. Experimental challenges in observing 2 to 3 decades of decay of $g^{(2)}(q, t)$ caused the slower decay to be overlooked previously.

Spectra of large spheres ( $d$ of $87,189,282,455 \mathrm{~nm}$ ) lack the prominent bimodal relaxation characteristic of small sphere spectra. However, fits of these spectra to eqs. 8 or 9 were unsatisfactory. rms fractional error for Eq. (10) was $1.5 \times 10^{-3}-4 \times 10^{-3}$. The stability of the fits was unsatisfactory, especially at elevated concentration and larger $d$. The best fits for $\theta$ and other parameters, plotted against $c$, were excessively scattered. Multiangle experiments were performed for $87 \mathrm{~nm}$ spheres in six solutions having $0 \leqslant c \leqslant 6$ $\mathrm{g} / \mathrm{l}$, and for $189 \mathrm{~nm}$ spheres in five solutions having $0 \leqslant c \leqslant 5$ $\mathrm{g} / \mathrm{l}$. Figure 2 shows the $q$-dependence of $\theta$ for $87 \mathrm{~nm}$ probes in $4 \mathrm{~g} / \mathrm{L} \mathrm{HPC}$ solution; $\theta$ corresponds to fits to eqs. 9,10 , and 11 (below). $\theta$ from Eq. 9 shows substantial deviations from a linear dependence on $q^{2}$ and has a substantial intercept as $q^{2} \rightarrow 0$. $\theta$ from fits to Eq. 10 has an irregular dependence on $q^{2}$. This lack of $q^{2}$-behavior is unreasonable for large spheres in dilute polymer solutions, because in such systems one expects the slowest mode to be diffusive, i.e., linear in $q^{2}$ with $\theta \rightarrow 0$ as $q^{2} \rightarrow 0$.

Because $\theta$ from Eq. 9 lacks a clear linear dependence on $q^{2}$, and because rms fractional errors of fits to Eq. 9 are not small, we conclude that Eq. 9 is unsatisfactory for large probes. Fits to Eq. 10 for concentrated HPC solutions lack stability, i.e., the outcome of the fit is sensitive to the initial guesses of the parameters; $\theta$ does not have a simple linear dependence $q^{2}$. We conclude that Eq. 10 is also unsatisfactory for large probes.

We observed, however, that $\beta$ increased with increasing $d$, and $\beta \approx 1$ at large $d$. Recalling that overparameterization causes nonlinear fits to be unstable, and that the large sphere spectra have fewer visible features than do small sphere spectra, we therefore tried another fitting function with fewer parameters, namely a fast stretched exponential and a slow pure exponential

$$
g^{(1)}(q, t)=A_{f} \exp \left(-\theta_{f} t^{\beta_{f}}\right)+\left(1-A_{f}\right) \exp (-\theta t) .
$$

Equation 11 gave fits with rms fractional errors $1 \times 10^{-3}-3.5 \times 10^{-3}$, i.e., fixing $\beta$ of Eq. 10 at $\beta=1$ did not increase the fitting errors. However, fits to Eq. 11 were very stable at all $c$, unlike fits with Eq. 10, in which the final fit

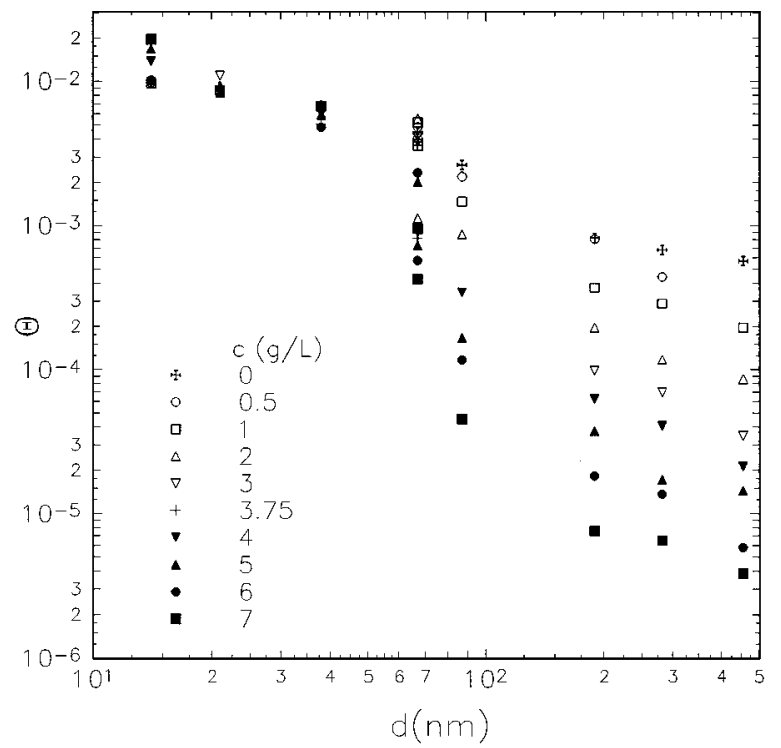

(a)

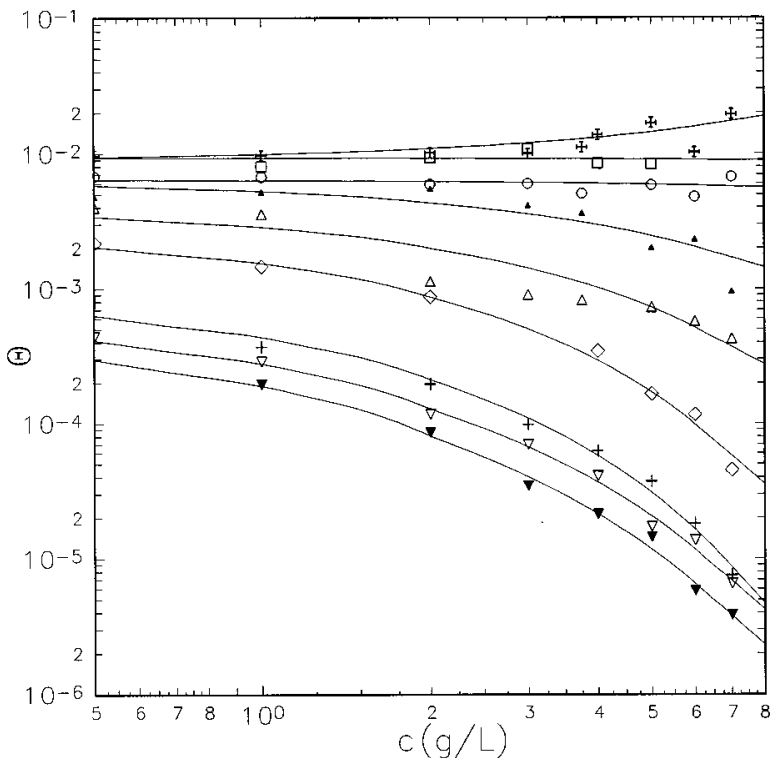

FIG. 3. Slow relaxation pseudorate $\theta$ (Eq. 10 for small spheres and Eq. 11 for large spheres), as functions of: (a) Probe diameter for different polymer concentrations; and (b) HPC concentration for small $14(\mathbf{W}), 21(\square), 38$ $(\bigcirc)$, and $67(\boldsymbol{\Delta}) \mathrm{nm}$ and large $67(\triangle), 87(\diamond), 189(+), 282(\nabla)$, and 455 $(\boldsymbol{\nabla}) \mathrm{nm}$ probes. Solid lines are stretched exponentials in $c$ with parameters in Table II. Units of $\theta$ are $(\mu S)^{-\beta}$. 
TABLE II. Concentration dependence of $\theta$, parameterized as $\theta=\theta_{0} \exp \left(-\alpha c^{\nu}\right)$ using $\theta$ from: (1) Eq. 10 for small spheres $(d \leqslant 67 \mathrm{~nm})$, and (2) Eq. 11 for large spheres $(d \geqslant 67 \mathrm{~nm})$.

\begin{tabular}{ccccc}
\hline \hline$d(\mathrm{~nm})$ & $\theta_{0}$ & $\alpha$ & $\nu$ & rms error \\
\hline 14 & $8.98 \times 10^{-3}$ & $-9.34 \times 10^{-2}$ & 1.0 & $1.51 \times 10^{-1}$ \\
21 & $9.43 \times 10^{-3}$ & $9.26 \times 10^{-3}$ & 0.97 & $9.43 \times 10^{-2}$ \\
38 & $6.46 \times 10^{-3}$ & $1.86 \times 10^{-2}$ & 0.98 & $1.05 \times 10^{-1}$ \\
67 & $6.41 \times 10^{-3}$ & 0.19 & 1.0 & $2.31 \times 10^{-1}$ \\
67 & & & & \\
87 & $4.09 \times 10^{-3}$ & 0.34 & 1.0 & $2.44 \times 10^{-1}$ \\
189 & $2.73 \times 10^{-3}$ & 0.55 & 1.0 & $1.16 \times 10^{-1}$ \\
282 & $9.35 \times 10^{-4}$ & 0.74 & 0.95 & $1.56 \times 10^{-1}$ \\
455 & $7.06 \times 10^{-4}$ & 0.94 & 0.82 & $9.86 \times 10^{-2}$ \\
\hline \hline
\end{tabular}

parameters were sensitive to the initial guesses. Furthermore, as seen in representative data in Figure 2, unlike $\theta$ from Eq. 10, $\theta$ from Eq. 11 shows diffusive $q^{2}$ behavior, as expected for large probes at long times. For large spheres, Eq. 10 is thus seen to overparameterize $g^{(1)}(q, t)$, therefore, Eq. 11 is the preferred form for $g^{(1)}(t)$.

Since Eq. 11 is simply Eq. 10 with $\beta=1$ forced, one might ask why a fit to Eq. 11 does not give precisely the same result as a fit to Eq. 10 , the fit to Eq. 10 giving $\beta \approx 1$. The answer is that fits of large sphere spectra to Eq. 10 do find that $\beta$ is close to 1.0. However, increasing the number of free parameters in the fit by floating $\beta$, rather than forcing $\beta=1$, increases the compliance of the fitting function, thereby increasing the errors in determining every parameter, without improving significantly the rms errors. Excessive compliance of the fitting function manifests itself as random scatter in all parameters (e.g., Figure 2, $\theta$ from Eq. 10), not just in the one "extra'" parameter. This random scatter disappears when $\beta=1$ is forced. In contrast, for small spheres, $\beta$ was needed as a free parameter. Fits of Eq. 11 to small sphere spectra gave much worse results than fits of Eq. 10.

Intermediate $(67 \mathrm{~nm})$ spheres show intermediate spectral behavior. Spheres having $d=67 \mathrm{~nm}$ do not show the evident bimodal relaxation that small spheres have; nor do fits of such spectra to Eq. 11 give better results than fits to Eq. 10 . Spectra of the $67 \mathrm{~nm}$ spheres were analyzed using both eqs. 10 and 11.

In summary, this section described functional forms that might describe our spectra. For small probes $(d<67 \mathrm{~nm})$, spectra are described well by Eq. 10, a sum of two stretched exponentials. For large spheres $(d>67 \mathrm{~nm})$, spectra are described well by Eq. 11, a sum of a fast stretched exponential and a slow pure-exponential. Spectra of the $67 \mathrm{~nm}$ spheres show transitional behavior. We are not claiming that eqs. 10 or 11 necessarily follow directly from a correct physical model; we are at this stage only claiming that eqs. 10 and 11 quantitatively parameterize $g^{(1)}(q, t)$.

\section{RESULTS}

In this section we present our detailed findings. A more generalized analysis appears in the Discussion. We determined $\theta, \beta, \theta_{f}, \beta_{f}$ and $A_{f}$ as described above; now we examine the concentration dependencies of these parameters. To anticipate our results: For each probe the slow relaxation pseudorate $\theta$ has a stretched exponential dependence on $c$, but $\theta$ has very different concentration dependencies for small and for large spheres. $\theta_{f}$ only depends weakly on $c ; \theta_{f} / \theta$ increases considerably with increasing probe size. The fraction $A_{f}$ of the fast decay rises with increasing $c$ over $0 \leqslant c \leqslant 4 \mathrm{~g} / \mathrm{L}$. At higher concentrations, $A_{f}$ for small spheres has a plateau, while for very large spheres, $A_{f}$ decreases above $c \approx 4 \mathrm{~g} / \mathrm{L}$. Finally, we consider the $q$-dependencies of the fitting parameters. For all probes, the slow relaxation is a diffusive, $q^{2}$-dependent mode. For large spheres, the fast relaxation has a more obscure $q$-dependence, which in some ranges of angles is linear in $q^{2}$. The fast mode of small spheres has a clear $q^{2}$-dependence at $c \leqslant 2 \mathrm{~g} / \mathrm{L}$, near$q^{2}$-scaling at $2 \leqslant c \leqslant 4 \mathrm{~g} / \mathrm{L}$ and large $q$, but has no simple $q$-dependence at $5 \mathrm{~g} / \mathrm{L}$.

Figures $3 \mathrm{a}$ and $3 \mathrm{~b}$ and Table II give $\theta$ of the slow decay for all probes and concentrations studied. Figure 3 a reveals how $\theta$ depends on $d$; Figure $3 \mathrm{~b}$ plots $\theta$ as a function of $c . \theta$ depends strongly on both variables. From Figure $3 \mathrm{a}$, depending on concentration, $\theta$ falls 20 to 1000 fold with a 30 fold increase of the probe diameter. The decrease in $\theta$ with increasing $d$ is larger in more concentrated solutions. In pure water $\theta$ decreases less than 20 fold over our range of $d$, while at $7 \mathrm{~g} / \mathrm{L} \theta$ of the same probes decreases approximately 1000 fold with increasing $d$ from 14 to $455 \mathrm{~nm}$. $\Delta \theta / \Delta d$ depends substantially on $d$, and at each $c$ is largest for intermediate $d$. For small spheres $(d \leqslant 38 \mathrm{~nm}), \theta$ decreases 1.5 to 2 fold with the 2.7 fold increase in $d$ from 14 to $38 \mathrm{~nm}$. For very large spheres $(d \geqslant 189 \mathrm{~nm}), \theta$ also decreases 1.8 to 2 fold with a 2.4 fold increase in $d$ from 189 to $455 \mathrm{~nm}$. However, for intermediate size probes $(38 \mathrm{~nm}<d<189 \mathrm{~nm}), \theta$ decreases drastically with increasing $d$. Depending on $c$, the 5 fold increase of probe diameter from 38 to $189 \mathrm{~nm}$ causes $\theta$ to decrease 10 to 500 fold.

From Figure $3 b$, there is a pronounced concentration dependence of $\theta$, but only at large $d$. For the $14 \mathrm{~nm}$ spheres, $\theta$ (Eq. 10) is constant up to $3 \mathrm{~g} / \mathrm{L}$, and perhaps increases at $c>3 \mathrm{~g} / \mathrm{L}$. $\theta$ of other small $(d<67 \mathrm{~nm})$ spheres shows no $c$-dependence. $67 \mathrm{~nm}$ spheres fall into a transition region between small and large $d$. The $c$-behavior of $\theta$ or $67 \mathrm{~nm}$ spheres depends on whether Eq. 10 or Eq. 11 is used to analyze $S(q, t)$. For large spheres $(d>67 \mathrm{~nm}), \theta$ falls 5 to 100 fold with increasing $c$; the extent of the fall increases with increasing probe size. 
For each probe diameter, we fit $\theta$ to

$$
\theta=\theta_{o} \exp \left(-\alpha c^{\nu}\right)
$$

using non-linear least-squares. ${ }^{25}$ Here $\alpha$ is the scaling prefactor, $\nu$ is the scaling exponent, and $\theta_{0}$ is the intercept. Fits to Eq. 12 are solid lines in Figure 3b; parameters appear in Table II. For small spheres, $\theta \approx \theta_{0}$. For large spheres, Eq. 12 works well. With increasing probe diameter, the intercept $\theta_{0}$ and the scaling exponent $\nu$ both decrease. For large spheres $(d \geqslant 87 \mathrm{~nm}), \alpha$ increases linearly with rising $d$, while for small spheres $(d \leqslant 38 \mathrm{~nm}) \alpha \approx 0$. Equation 12 does not work as well for $67 \mathrm{~nm}$ spheres as for other spheres.

Figures $4 a-4 b$ show representative data on the $q$-dependence of $\theta$. Figure 4 a shows $\theta$ from Eq. 10, for the small $21 \mathrm{~nm}$ spheres at 1,2 and $4 \mathrm{~g} / \mathrm{L}$. At each concentration, $\theta$ increases approximately 7 to 10 fold from the smallest to

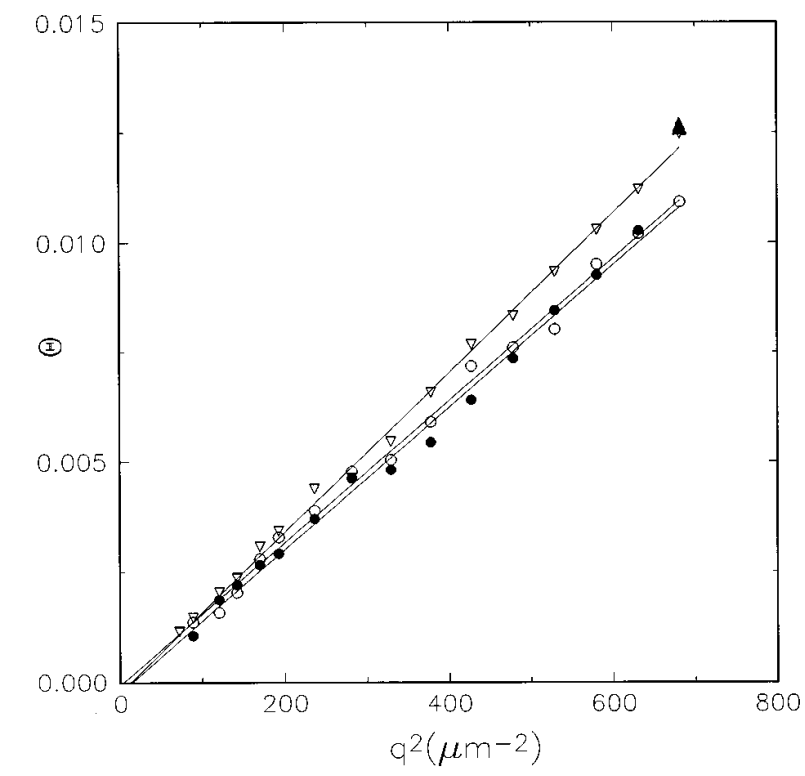

(a)

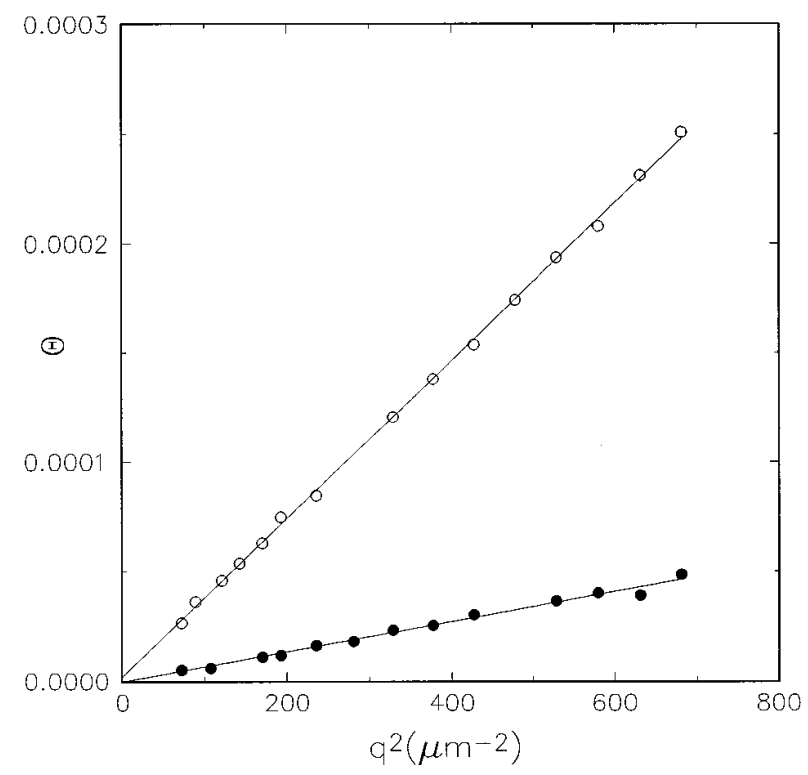

(b)

FIG. 4. Slow relaxation pseudorate $\theta$ as a function of $q^{2}$ for probes in HPC: water: (a) $21 \mathrm{~nm}$ probes in $1 \mathrm{~g} / \mathrm{L}(\bigcirc), 2 \mathrm{~g} / \mathrm{L}(\nabla)$, and $4 \mathrm{~g} / \mathrm{L}(\bullet)$; (b) 189 $\mathrm{nm}$ probes in $2 \mathrm{~g} / \mathrm{L}(\bigcirc)$ and $5 \mathrm{~g} / \mathrm{L}(\bullet)$. Solid lines are best linear fits. Points $(\boldsymbol{\Delta})$ on Figure $4 \mathrm{a}$ were omitted from the fit. Units of $\theta$ are $(\mu S)^{-\beta}$.

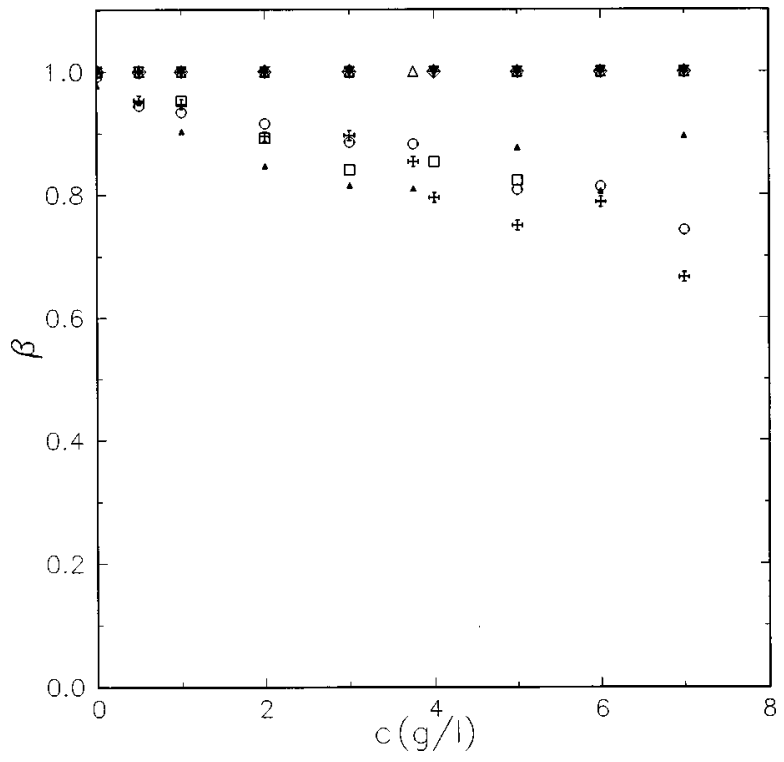

FIG. 5. Concentration dependence of $\beta$ (Eq. 10), for spheres of nominal diameter $14(\mathbf{W}), 21(\square), 38(\bigcirc)$, and $67(\boldsymbol{\Lambda}) \mathrm{nm}$. For large spheres, from Eq. $11 \beta=1$ (all other symbols).

the largest $q$. At all HPC concentrations, $\theta$ shows clearly diffusive behavior, i.e., $\theta \sim a q^{2}$ with zero intercept as $q \rightarrow 0$. Figure $4 \mathrm{~b}$ gives the $q$-dependence of $\theta$ from Eq. 11 for 189 $\mathrm{nm}$ spheres at HPC concentrations of 2 and $5 \mathrm{~g} / \mathrm{L}$. The $q$-dependence of $\theta$ for $87 \mathrm{~nm}$ spheres is identical to Figure 4 b. For all large spheres, $\theta$ has a strong concentration dependence. For both 87 and $189 \mathrm{~nm}$ probes, $\theta$ shows clear diffusive behavior ( $\theta \sim a q^{2}$ with no $q^{2} \rightarrow 0$ intercept) at all HPC concentrations.

Figure 5 gives the stretching exponent $\beta$ of the slow relaxation as a function of HPC concentration. For small spheres, $\beta$ from Eq. 10 falls with increasing $c$, from almost 1.0 at zero concentration to 0.7 (for 14 and $38 \mathrm{~nm}$ probes) at $7 \mathrm{~g} / \mathrm{L}$ or 0.8 (for $21 \mathrm{~nm}$ probes at $5 \mathrm{~g} / \mathrm{L}$ ). The decrease in $\beta$ with increasing $c$ is nearly monotonic for all small probes. For $67 \mathrm{~nm}$ probes, $\beta$ is more scattered than $\beta$ for smaller spheres; $\beta$ decreases with rising $c$ at $c<4 \mathrm{~g} / \mathrm{L}$, but is approximately constant for $c>4 \mathrm{~g} / \mathrm{L}$. Figure 6 shows exemplary $q$-dependencies of $\beta$ (Eq. 10) for $21 \mathrm{~nm}$ spheres in solutions of 1,2 and $4 \mathrm{~g} / \mathrm{L}$. $\beta$ decreases with rising $c$, but decreases at most weakly with increasing $q$. Spectra of large spheres have $\beta \equiv 1$ for all $c$ and $q$.

Figure 7 shows the fast decay pseudorate $\theta_{f}$ (units $(\mu S)^{-\beta_{f}}$ ) as functions of $c$ and $d$. Regardless of the probe diameter, $\theta_{f}$ increases (Figure $7 \mathrm{a}$ ) by not more than $50 \%$ with increasing $c$. At all $c$, larger probes generally have a smaller $\theta_{f}$. Quantitatively, our probe diameters $d$ have a 30 fold range, but the variation in $\theta_{f}$ with $d$ is less than 12 fold. Figure $7 \mathrm{~b}$ shows explicitly the probe size dependence of $\theta_{f}$. For small spheres $(d \leqslant 38 \mathrm{~nm}), \theta_{f}$ is practically independent of $d$. On increasing the probe diameter further, from 38 to $455 \mathrm{~nm}, \theta_{f}$ monotonically decreases by an order of magnitude. Above $d=150 \mathrm{~nm}, \theta_{f}$ is nearly independent of $d$.

Figure 8 gives the $q$-dependence of $\theta_{f}$. Figure $8 \mathrm{a}$ describes $21 \mathrm{~nm}$ spheres. At $c \leqslant 2 \mathrm{~g} / \mathrm{L}, \theta_{f}$ increases 12 to 15 fold from smallest to largest $q$, while $\theta_{f}$ can be approximated 


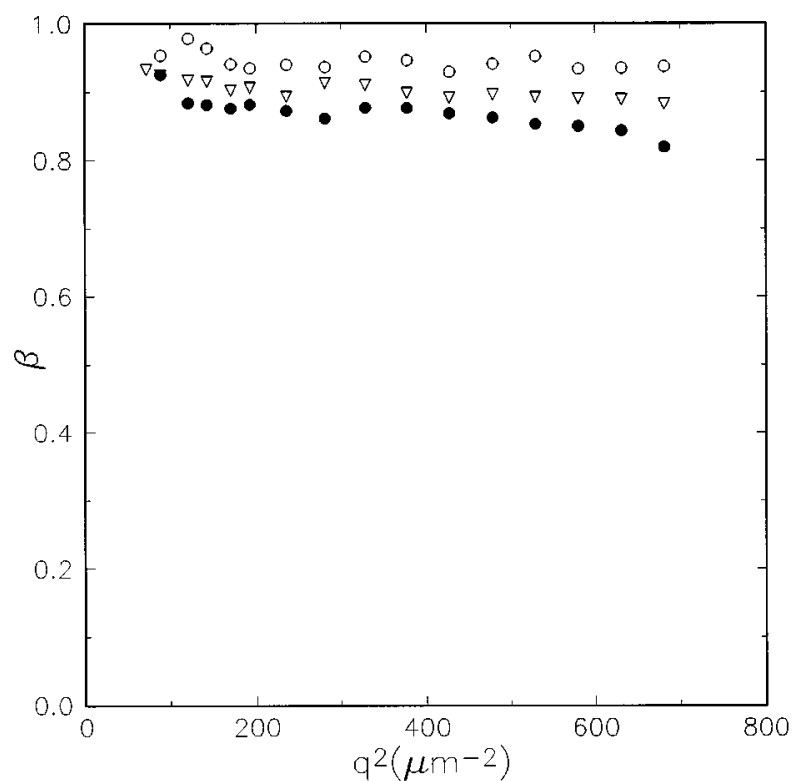

FIG. 6. Stretching exponent $\beta$ (from Eq. 10) as a function of $q^{2}$ for $21 \mathrm{~nm}$ probes in $1 \mathrm{~g} / \mathrm{L}(\bigcirc), 2 \mathrm{~g} / \mathrm{L}(\nabla)$, and $4 \mathrm{~g} / \mathrm{L}(\bullet)$ HPC solution.

by two linear dependencies, namely $\theta_{f} \approx a_{0} q^{2}$ at large $q$, and $\theta_{f} \approx a_{1} q^{2}+b$ with small intercept $b$ at small $q$; one finds $a_{1}<a_{0}$. At larger $c(2<c \leqslant 4 \mathrm{~g} / \mathrm{L}), \theta_{f}$ is more scattered, but increases about 30 fold from smallest to largest $q$. The $q$-dependence of $\theta_{f}$ at $2<c \leqslant 4 \mathrm{~g} / \mathrm{L}$ is the same as its $q$-dependence at $c \leqslant 2 \mathrm{~g} / \mathrm{L}$. The concentration dependence of $\theta_{f}$ falls into two regimes, depending on $q$. At small $q, \theta_{f}$ decreases with rising $c$; the slope $a$ is nearly independent of $c$. At large $q, \theta_{f}$ is much more scattered; its $c$-dependence is unclear. At the highest concentration studied $(5 \mathrm{~g} / \mathrm{L}), \theta_{f}$ is strongly scattered with no clear $q$-dependence.

Figures $8 \mathrm{~b}$ and $8 \mathrm{c}$ give the $q$-dependencies of $\theta_{f}$ (Eq. 11) for large ( 87 and $189 \mathrm{~nm})$ spheres at several HPC concentrations. $\theta_{f}$ of the 87 and $189 \mathrm{~nm}$ spheres does not show a strong $c$-dependence, but increases with rising $q$. Specifically: First, consider the $87 \mathrm{~nm}$ spheres. At $c \leqslant 4 \mathrm{~g} / \mathrm{L}$, $\theta_{f}$ is scattered but increases with increasing $q^{2}$. At $c>4 \mathrm{~g} / \mathrm{L}$, $\theta_{f}$ is much less scattered than at $c<4 \mathrm{~g} / \mathrm{L}$. At 5 and $6 \mathrm{~g} / \mathrm{L}$, $\theta_{f}$ shows two regimes of $q$-behavior: (1) at $q^{2} \leqslant 2.3 \times 10^{-10}$ $\mathrm{m}^{-2}, \theta_{f}$ rises 2 to 3 fold and follows $a q^{2}+b$ with a small intercept $b$; (2) at $q^{2}>2.3 \times 10^{-10} \mathrm{~m}^{-2}, \theta_{f}$ has a nearplateau. Second, consider the $189 \mathrm{~nm}$ spheres. $\theta_{f}$ is largely independent of $c$ and (at small $q$ ) of $q$; at larger $q, \theta_{f}$ has a linear dependence $\theta_{f} \sim a q^{2}+b$ with non-zero intercept $\mathrm{b}$.

To summarize, for all probe sizes and almost every concentration studied $\theta_{f}$ often showed linear dependence on $q^{2}$ at some (usually low) $q$, sometimes with a very small slope. The $q^{2} \rightarrow 0$ intercept of $\theta_{f}$ is sometimes non-zero.

Figure 9 presents the concentration dependence of $\beta_{f}$. For small probes, $\beta_{f}$ is substantially scattered but falls with increasing $c$, from $0.5-0.6$ at $1 \mathrm{~g} / \mathrm{L}$ to $0.2-0.3$ at $7 \mathrm{~g} / \mathrm{L}$. $\beta_{f}$ is more scattered at $c<1 \mathrm{~g} / \mathrm{L}$ than at $c>1 \mathrm{~g} / \mathrm{L}$. For small spheres: Within experimental error, larger probes have a larger $\beta_{f}$. For large spheres at $c<1 \mathrm{~g} / \mathrm{L}, \beta_{f}$ (Eq. 11) varies substantially for probes of different $d ; \beta_{f}$ is less scattered for $c \geqslant 1 \mathrm{~g} / \mathrm{L}$. $\beta_{f}$ falls from $0.75-0.87$ at $1 \mathrm{~g} / \mathrm{L}$ to $0.5-0.6$ and (a)
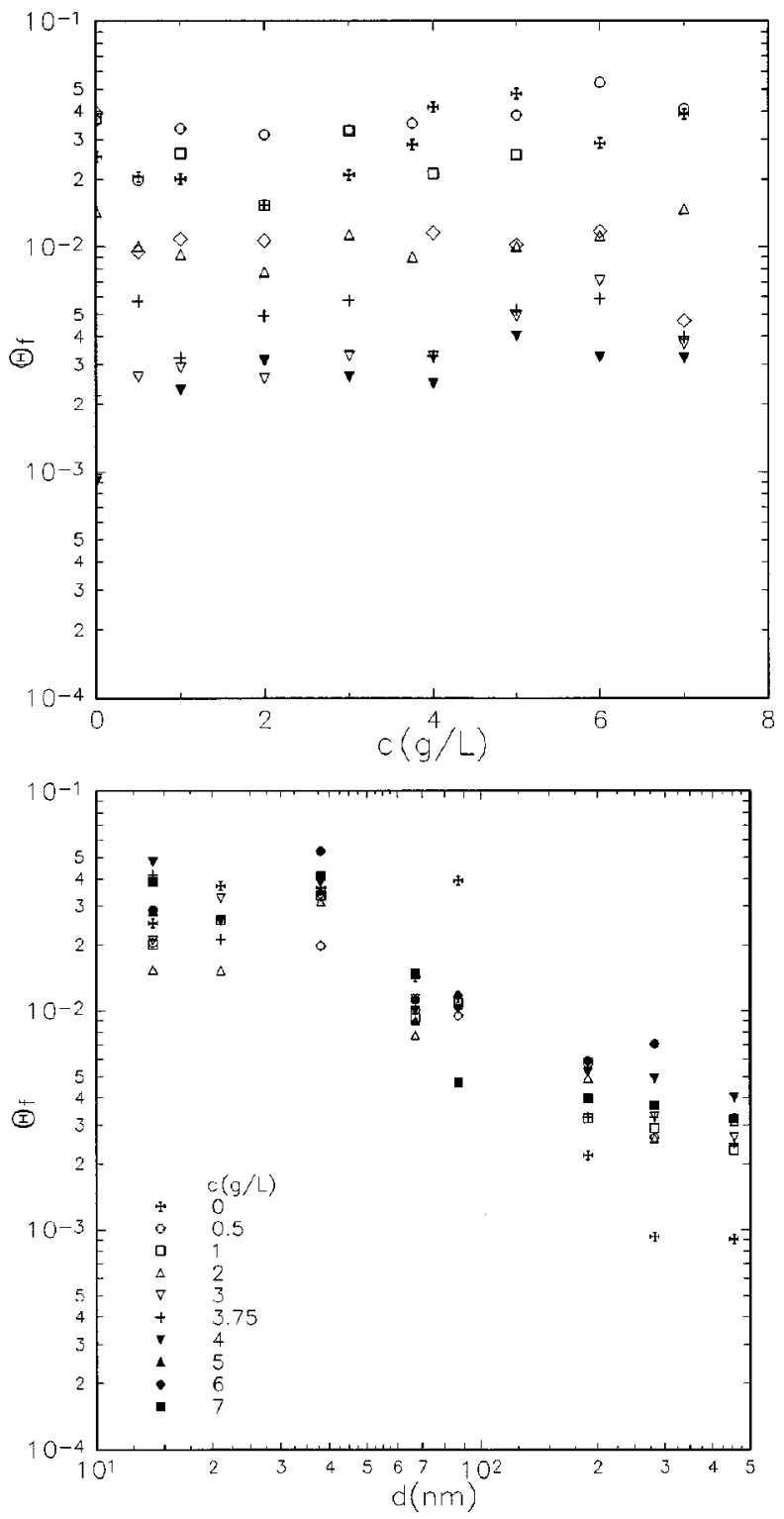

FIG. 7. Fast relaxation pseudorate $\theta_{f}$ (Eq. 10 for small spheres and Eq. 11 for large spheres) as functions of (a) HPC concentration, for small 14 ( $21(\square), 38(\bigcirc) \mathrm{nm}$, and large $67(\triangle), 87(\diamond), 189(+), 282(\nabla)$, and 455 $(\boldsymbol{\nabla}) \mathrm{nm}$ probes; and (b) probe diameter. Units of $\theta$ are $(\mu S)^{-\beta_{f}}$.

apparent saturation near the solutionlike-meltlike transition at $c^{+} \approx 6 \mathrm{~g} / \mathrm{L}$. At each $c, \beta_{f}$ is consistently larger for large spheres than small spheres. For $d>67 \mathrm{~nm}$ the dependence of $\beta_{f}$ on $d$ is weak.

Figures $10 \mathrm{a}-10 \mathrm{~b}$ give representative $q$-dependencies of $\beta_{f}$. For $21 \mathrm{~nm}$ spheres (Figure 10a), at $1 \mathrm{~g} / \mathrm{L}, \beta_{f} \approx 0.6$ is $q$-independent; at higher polymer concentrations, at small $q$, $\beta_{f}$ monotonically decreases with increasing $q$, while at large $q, \beta_{f}$ is $q$-independent. For large $189 \mathrm{~nm}$ spheres (Figure $10 \mathrm{~b}), \beta_{f}$ decreases with increasing $c$ and increases slightly with increasing $q$. For $87 \mathrm{~nm}$ spheres, at all $c$, $q$-dependencies of $\beta_{f}$ are very similar to $q$-dependencies of $189 \mathrm{~nm}$ spheres. In general, we found that the $q$-dependence of $\beta_{f}$ is weaker for larger concentrations and larger probes.

Figures 11 presents the $c$-dependence of $A_{f}$, for small $(d<67 \mathrm{~nm})$ and intermediate $(67$ and $87 \mathrm{~nm})$ spheres (Figure 11a), and large $(d>87 \mathrm{~nm})$ spheres (Figure 11b). For small 
(a)

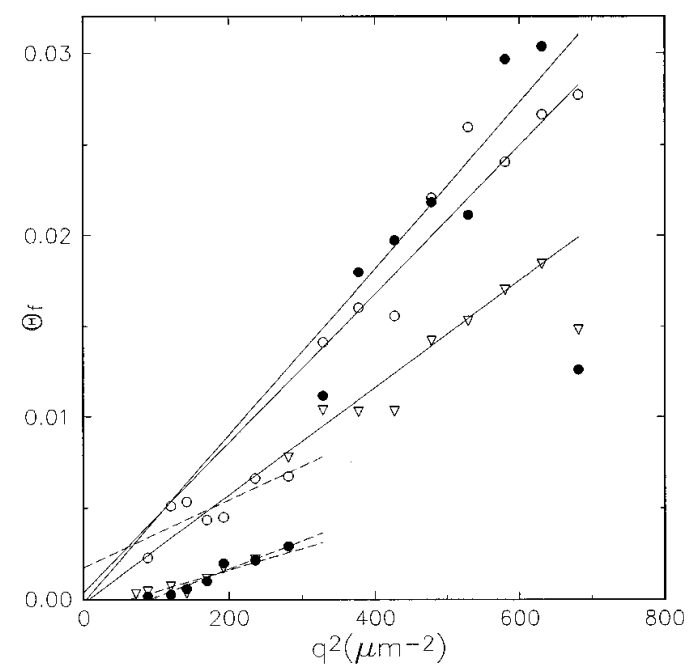

(b)

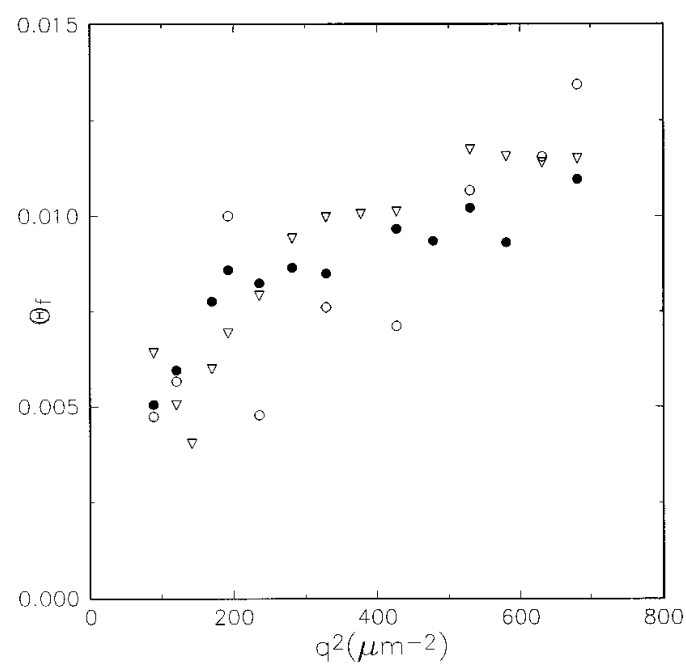

(c)

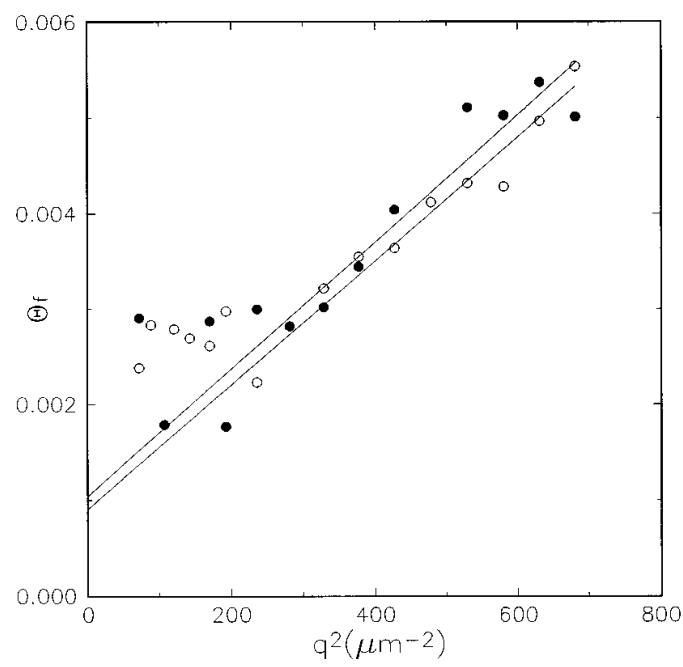

FIG. 8. Fast decay pseudorate $\theta_{f}$ as a function of $q^{2}$ for small (Eq. 10) and large (Eq. 11) probes. (a) $21 \mathrm{~nm}$ spheres in $1 \mathrm{~g} / \mathrm{L}(O), 2 \mathrm{~g} / \mathrm{L}(\nabla)$, and $4 \mathrm{~g} / \mathrm{L}$ (๑) HPC: water (solid and dashed lines represent linear fits for high and low q respectively); (b) $87 \mathrm{~nm}$ spheres in $2 \mathrm{~g} / \mathrm{L}(\bigcirc), 5 \mathrm{~g} / \mathrm{L}(\bullet)$, and $6 \mathrm{~g} / \mathrm{L}$ $(\nabla)$ HPC: water; (c) $189 \mathrm{~nm}$ spheres in $2 \mathrm{~g} / \mathrm{L}(\bigcirc)$ and $5 \mathrm{~g} / \mathrm{L}(\bullet)$ HPC: water (solid lines represent linear fits for high q). Units of $\theta$ are $(\mu S)^{-\beta_{f}}$.

spheres, $A_{f}$ (Eq. 10) monotonically rises with increasing $c$, from $0.03-0.08$ at zero concentration to $0.2-0.3$ at $4 \mathrm{~g} / \mathrm{L}$ and then tends to plateau at higher $c$. In general, smaller probes tend to have smaller $A_{f}$ values. For intermediate $67 \mathrm{~nm}$

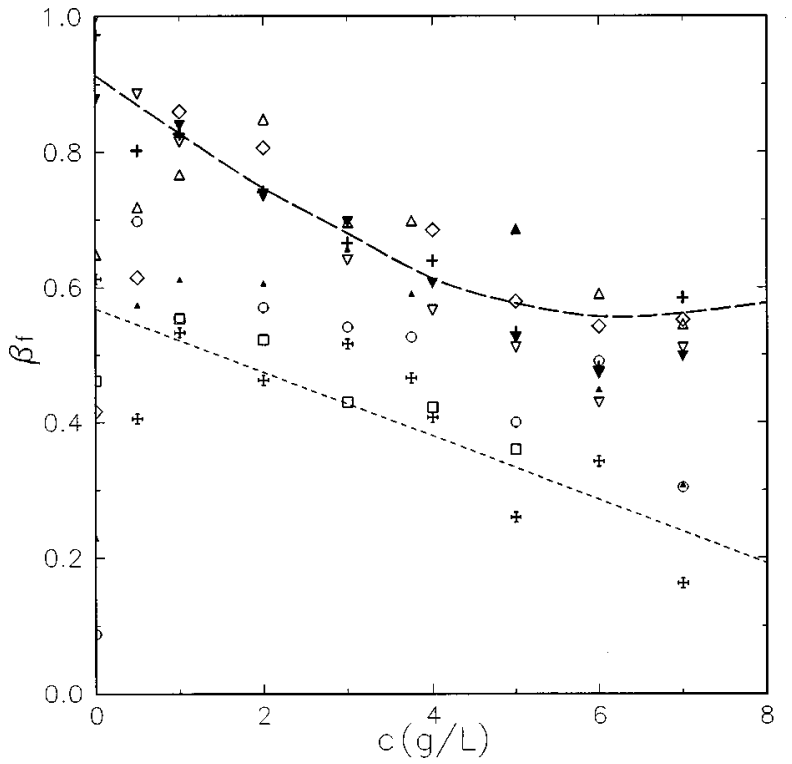

FIG. 9. Stretching exponent $\beta_{f}$, as a function of concentration, for small spheres of nominal diameter $14(\boldsymbol{\Psi}), 21(\square), 38(\bigcirc)$, and $67(\mathbf{\Lambda}) \mathrm{nm}$, using Eq. 10, and large probes of nominal diameter $67(\triangle), 87(\diamond), 189(+)$, $282(\nabla)$, and $455(\boldsymbol{\nabla}) \mathrm{nm}$, using Eq. 11. Dashed lines are drawn to guide the eye.

spheres, $A_{f}$ from Eq. 10 is small $\left(A_{f}<0.05\right)$ and almost $c$-independent up to $4 \mathrm{~g} / \mathrm{L}$. At higher $c, A_{f}$ rises to $0.3-0.4$. For $67 \mathrm{~nm}$ probes, $A_{f}$ from Eq. 11 rises very quickly from 0.12 at zero concentration to 0.6 at $2 \mathrm{~g} / \mathrm{L}$. At larger $c, A_{f}$ is constant with an average value of 0.65 . For $87 \mathrm{~nm}$ probes, $A_{f}$ (Eq. 11) increases almost monotonically from 0.05 at zero concentration to 0.62 at $7 \mathrm{~g} / \mathrm{L}$. Figure $11 \mathrm{~b}$ shows $A_{f}$ for large probes. $A_{f}$ (Eq. 11) increases from 0.2 to $0.5-0.6$ as $c$ rises from 0 to $4 \mathrm{~g} / \mathrm{L}$. Above $4 \mathrm{~g} / \mathrm{L} \mathrm{HPC}, A_{f}$ decreases to $0.2-0.33$ at $7 \mathrm{~g} / \mathrm{L}$.

Figures 12 give examples of the $q$-dependence of $A_{f}$. Figure 12a shows $A_{f}$ for $21 \mathrm{~nm}$ spheres; $A_{f}$ (Eq. 10) decreases quasi-exponentially with increasing $q^{2}$. At very large $q^{2}, A_{f}$ is approximately $0.2-0.25$ for all concentrations studied. Figure 12b gives $A_{f}$ (Eq. 11) for the $189 \mathrm{~nm}$ spheres. For $189 \mathrm{~nm}$ spheres, $A_{f}$ is much more scattered at small than large $c$. The $q$-dependencies of $A_{f}$ for the 87 (not shown) and $189 \mathrm{~nm}$ spheres are different at small $c$, but are very similar at large $c$. For $189 \mathrm{~nm}$ probes (Figure 12b), in $2 \mathrm{~g} / \mathrm{L}$ HPC, $A_{f}$ increases about 2 fold with rising $q$ at low $q$, but is $q$-independent at $q^{2}>3 \times 10^{-10} \mathrm{~m}^{-2}$. At $5 \mathrm{~g} / \mathrm{L}, A_{f}$ increases monotonically from 0.4 to 0.6 as $q$ increases. For $87 \mathrm{~nm}$ spheres (not shown), at low $c, A_{f}$ is almost $q$-independent, while at high $c, A_{f}$ increases about 1.5 fold from the smallest to the largest $q$. In all systems at fixed $q, A_{f}$ generally increases with increasing $c$. This behavior is shown on Figure 11 for $90^{\circ}$ scattering. The behavior is in fact the same at all angles, as may be seen from the Figure 12 .

Figure 13 shows the $c$-dependence of $\theta \eta . \eta$ is from Phillies and Quinlan. ${ }^{6}$ For small spheres $(d<40 \mathrm{~nm}), \theta$ does not track $\eta^{-1}$, so $\theta \eta$ increases 150 to 400 fold as $c$ increases from 0 to $7 \mathrm{~g} / \mathrm{L}$. For intermediate size spheres $(67,87 \mathrm{~nm})$, $\theta \eta$ increases 6 to 20 fold with rising $c$. For large spheres 


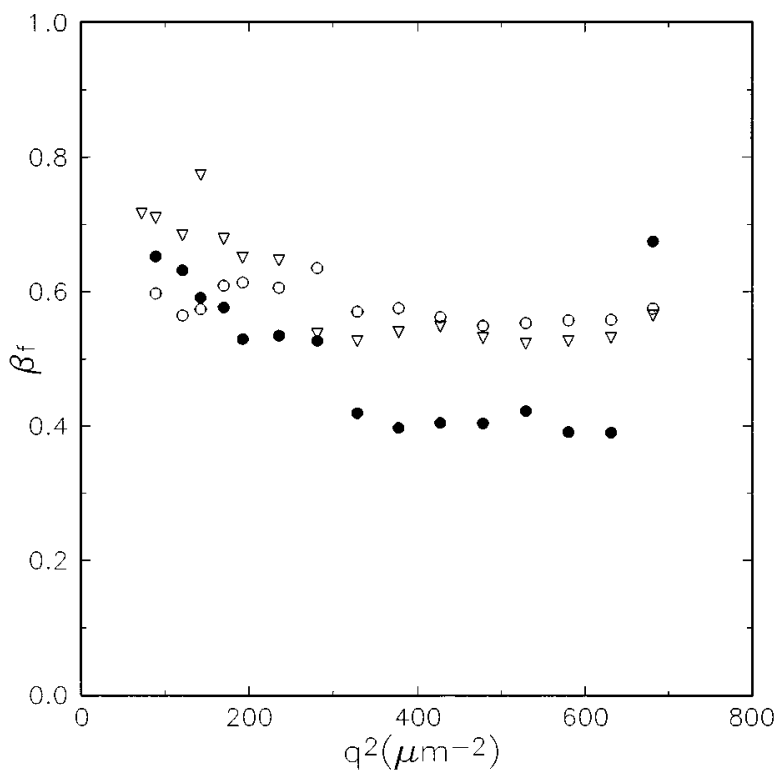

(a)
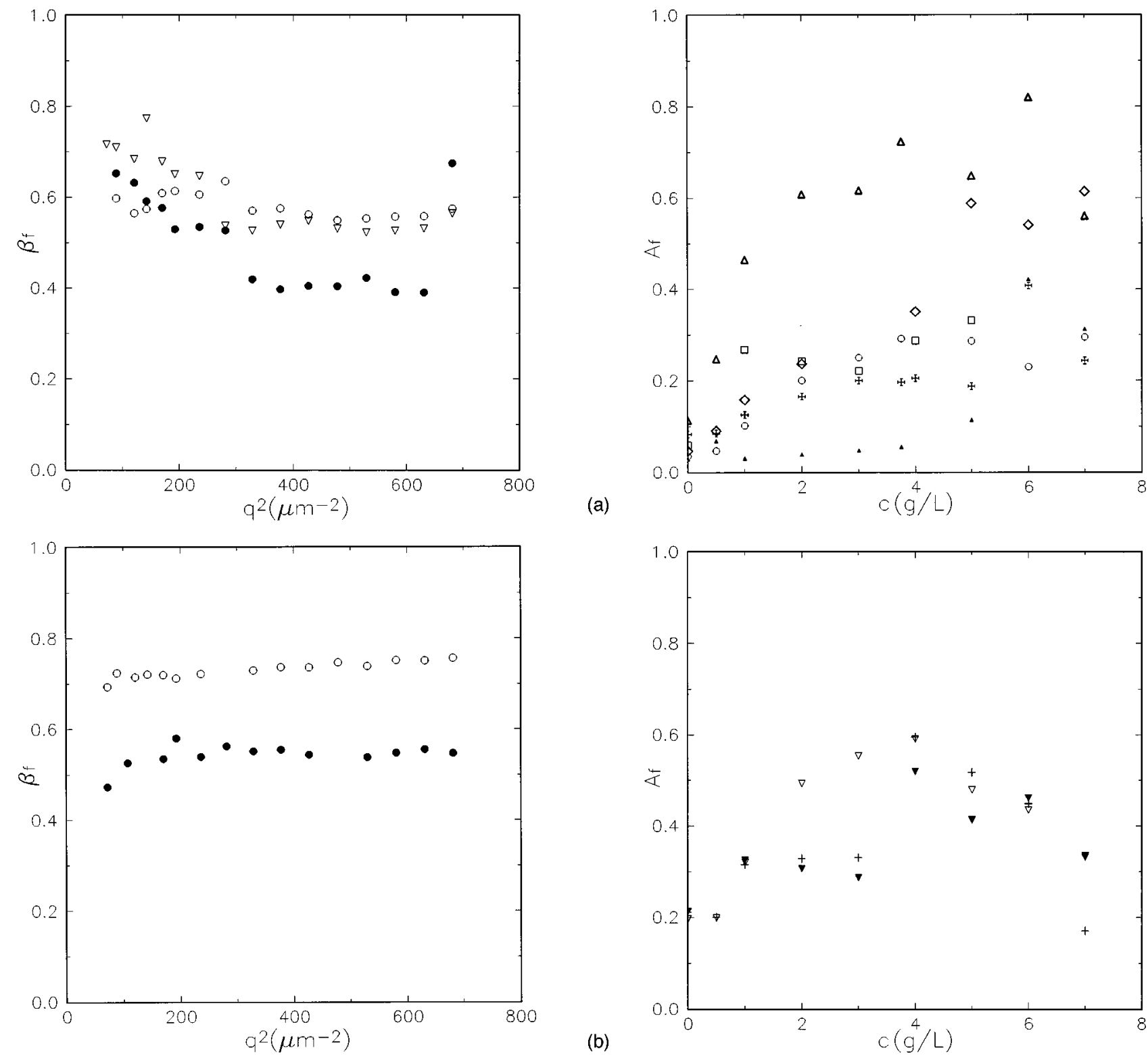

FIG. 10. Stretching exponent $\beta_{f}$ of the fast relaxation from Eq. 10 (small spheres) and from Eq. 11 (large spheres) for: (a) $21 \mathrm{~nm}$ probes in $1 \mathrm{~g} / \mathrm{L}(\bigcirc)$, $2 \mathrm{~g} / \mathrm{L}(\nabla)$, and $4 \mathrm{~g} / \mathrm{L}(\bullet)$ HPC: water; (b) $189 \mathrm{~nm}$ probes in $2 \mathrm{~g} / \mathrm{L}(\bigcirc)$ and $5 \mathrm{~g} / \mathrm{L}(\bullet)$ HPC: water.

( $d>100 \mathrm{~nm}), \theta \eta$ is almost constant, but fluctuates within 2 fold of its average value.

From Figure 3, $\theta$ follows Eq. 12, a stretched exponential in $c$. Figure 14 gives the scaling pre-factor $\alpha$ of Eq. 12 as a function of probe diameter. For $d \leqslant 67 \mathrm{~nm}, \alpha$ increases 50 fold with increasing $d$. For $d>67 \mathrm{~nm}, \alpha$ increases only 10 to 15 fold as $d$ increases from 87 to $455 \mathrm{~nm}$. For large probes, one finds $\alpha \sim \sqrt{d}$ (solid line, Figure 14). The dashed line is the viscosity pre-factor $\alpha_{\nu} \approx 0.97$ from $\eta=\eta_{0} \exp \left(\alpha_{\nu} c^{\nu}\right)$ for $\eta$ of $1 \mathrm{MDa}$ HPC. ${ }^{6}$ Overall, $\alpha$ increases with increasing $d$, saturating at large $d$ to $\alpha \approx \alpha_{\nu}$.

\section{DISCUSSION}

Our spectra uniformly fit to

$$
g^{(1)}(t)=\left(1-A_{f}\right) \exp \left(-\theta t^{\beta}\right)+A_{f} \exp \left(-\theta_{f} t^{\beta_{f}}\right) .
$$

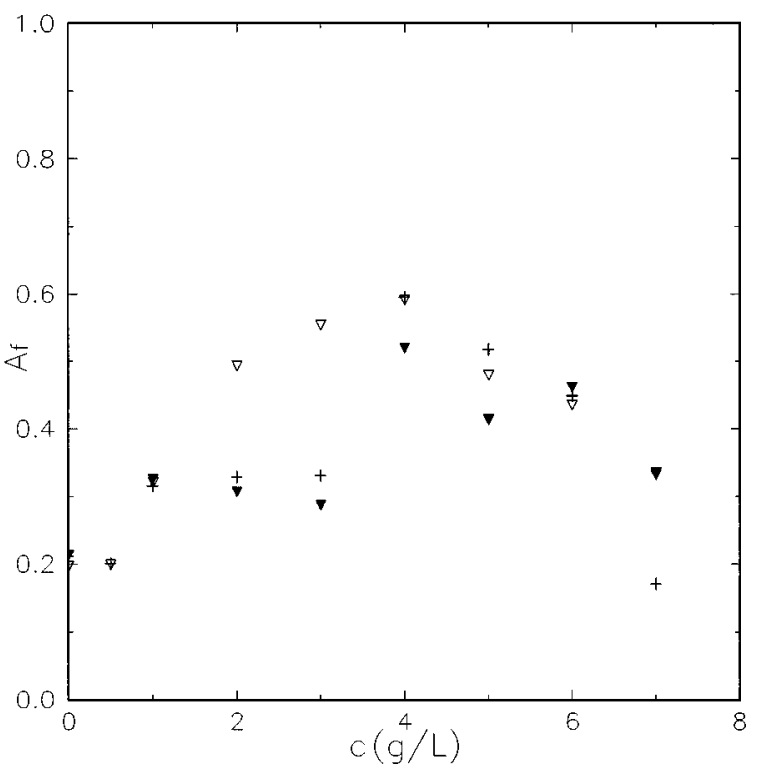

FIG. 11. Amplitude fraction parameter $A_{f}$ of the fast mode (Eq. 10 for small spheres $(d \leqslant 67 \mathrm{~nm})$ and Eq. 11 for large spheres $(d \geqslant 67 \mathrm{~nm}))$ as a function of HPC concentration for: (a) small $14(\mathbf{W}), 21(\square), 38(\bigcirc)$, and $67(\mathbf{\Delta})$ $\mathrm{nm}$, and intermediate $67(\triangle), 87(\diamond) \mathrm{nm}$; (b) very large $189(+), 282(\nabla)$, and $455(\boldsymbol{\nabla}) \mathrm{nm}$ probes.

$\beta$ has a strong probe size dependence, being $\approx 1$ for large probes and $<1$ for smaller probes. One underlying function, Eq. 13, thus fits all spectra; for large spheres, the constraint $\beta=1$ improved the stability of the fitting process without reducing the accuracy of the fit.

Our analysis decomposes spectra of polystyrene sphere probes into two modes, a fast stretched-exponential mode and a slower exponential or stretched-exponential mode. It should be emphasized that our interpretation of the modes as stretched exponentials is phenomenological. A group of exponential modes whose sum approximates a stretchedexponential decay cannot, within the limits of our experimental method, be distinguished from a single stretchedexponential mode. Our remarks on the properties of single modes may therefore actually be statements about the aggre- 
(a)

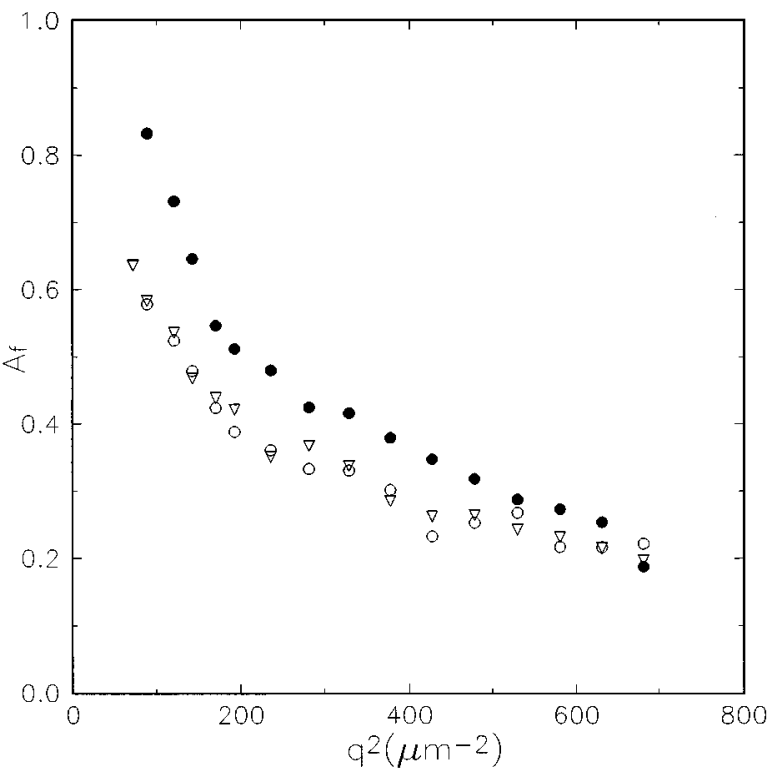

(b)

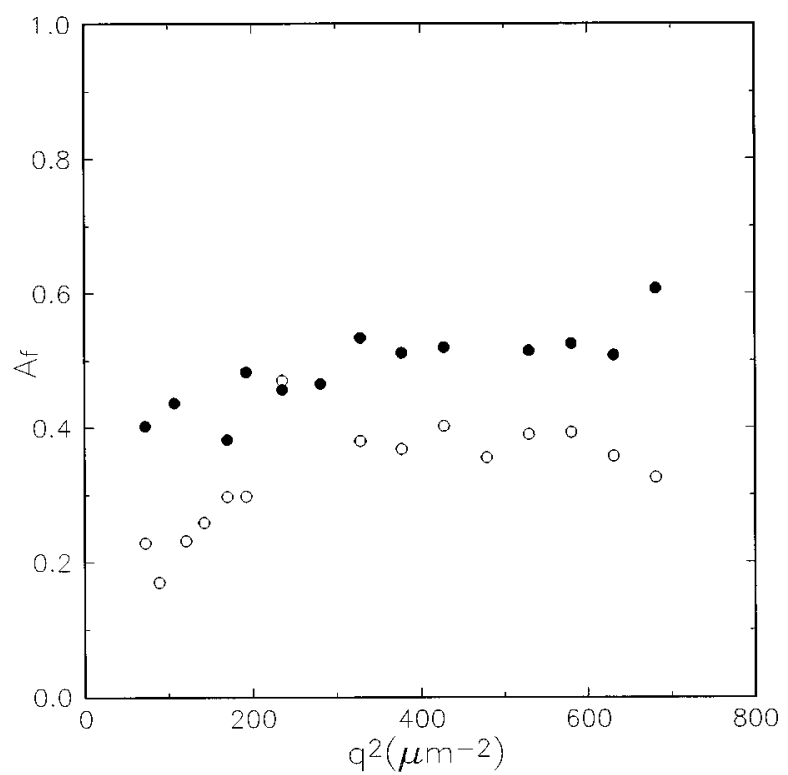

FIG. 12. Amplitude fraction parameter $A_{f}$ of the fast mode from Eq. 10 (small spheres) and from Eq. 11 (large spheres) as a function of $q^{2}$ for: (a) $21 \mathrm{~nm}$ probes in $1 \mathrm{~g} / \mathrm{L}(\bigcirc), 2 \mathrm{~g} / \mathrm{L}(\nabla)$, and $4 \mathrm{~g} / \mathrm{L}(\bullet)$ HPC: water; (b) 189 $\mathrm{nm}$ spheres in $2 \mathrm{~g} / \mathrm{L}(\bigcirc)$ and $5 \mathrm{~g} / \mathrm{L}(\bullet)$ HPC: water.

gate behavior of a group of modes. However, sums of two pure exponentials do not fit our spectra. A single stretchedexponential mode may be a stretched exponential or an aggregate of pure exponentials, but is observably not a single pure exponential.

Our results may be divided naturally into three regimes, namely those for small $(d<40 \mathrm{~nm})$, intermediate (67 and 87 $\mathrm{nm})$, and large $(d>100 \mathrm{~nm})$ probes. In each regime we observe a fast and a slow decay mode. By "slow" and "fast" we refer to relative values of $\theta$ and $\theta_{f}$, which usually differ by 5 to 100 fold in our units. In some cases (small spheres) in which $\theta_{f} / \theta$ is only $1.1-3.0, \beta$ and $\beta_{f}$ are very different, namely $\beta=0.7-1.0$ and $\beta_{f}=0.2-0.6$, so the modes are still readily separated.

In our results here, $\beta$ and $\beta_{f}$ are both in the range $(0,1)$. For large spheres, $\beta \approx 1.0$; for small spheres, $\beta$ falls with

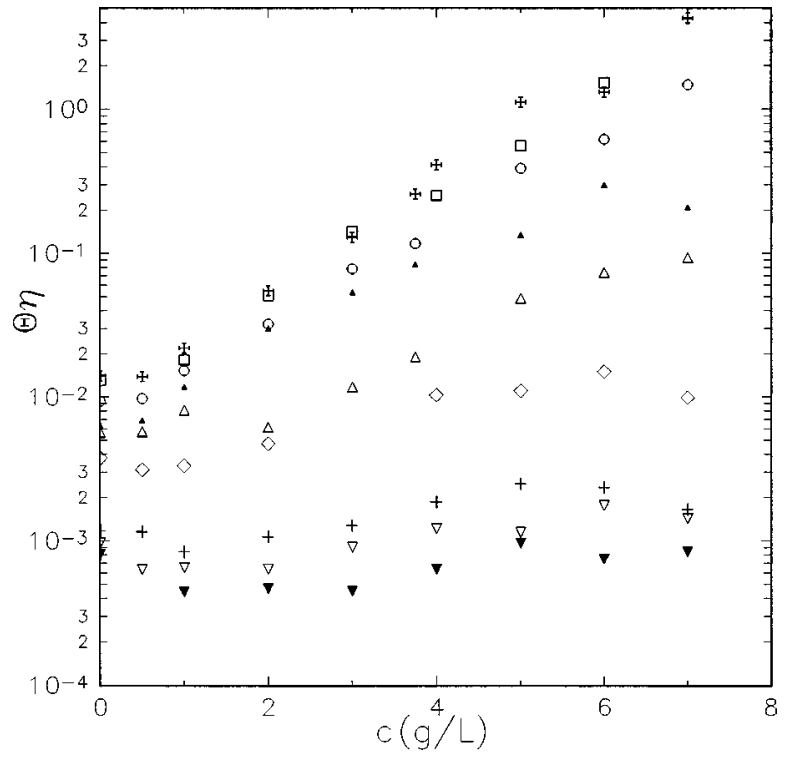

FIG. 13. Concentration dependence of the product of slow relaxation pseudorate $\theta$ and solution viscosity $\eta$ for small $14(\mathbf{W}), 21(\square), 38(\bigcirc), 67(\mathbf{A})$ $\mathrm{nm}$, and large $67(\triangle), 87(\diamond), 189(+), 282(\nabla)$, and $455(\boldsymbol{\nabla}) \mathrm{nm}$ probes. $\theta$ is from Eq. 10 for small spheres and Eq. 11 for large spheres. $\eta$ is from Ref. 6.

increasing concentration, but is nearly independent of $q^{2}$. The exponent $\beta_{f}$ is consistently less than $\beta$. For all sphere sizes, $\beta_{f}$ falls with increasing concentration. For large spheres, $\beta_{f}$ is independent of $q^{2}$. For small spheres, $\beta_{f}$ falls within increasing $q^{2}$, especially at small $q^{2}$.

For a monodisperse suspension of spherical probes in a simple solvent, the field correlation function is $\exp \left(-D q^{2} t\right)$, which is analogous to Eq. 13 if $\beta=1$ and if one identifies $\theta=D q^{2}$. In our systems, decay modes were often stretched rather than simple exponentials, so $D q^{2}$ and $\theta$ fundamentally

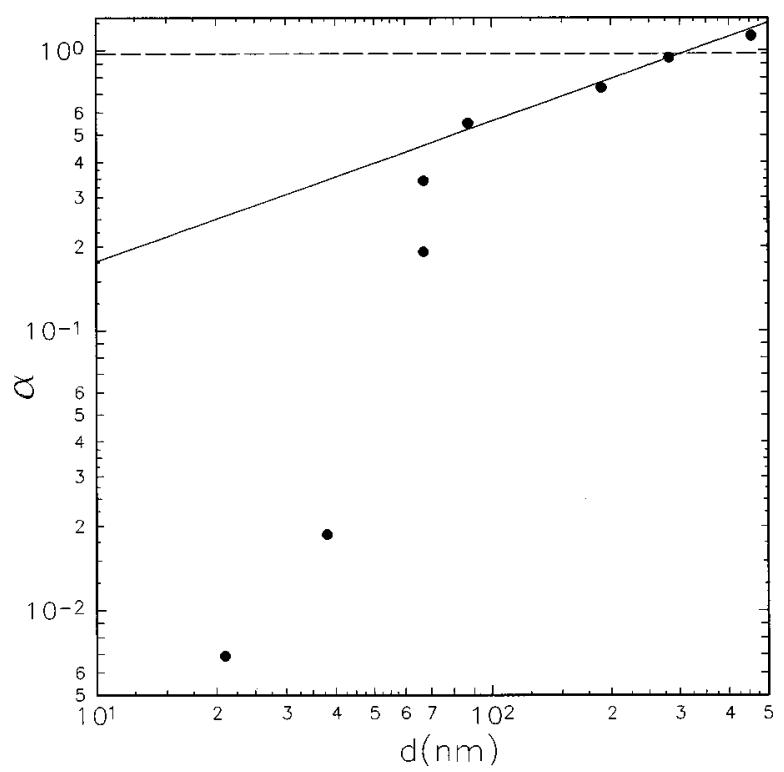

FIG. 14. Scaling pre-factor $\alpha$ from the fits to Eq. 12 as a function of probe diameter $d$. A solid line of slope $1 / 2$ is displayed for reference. Dashed line represents $\alpha_{\nu}=0.97$ for $\eta$ of 1 MDa HPC (Ref. 6). 
differ: $D q^{2}$ is a true decay rate, with units $t^{-1}$, while $\theta$ is a pseudorate whose units are not $t^{-1}$. Nonetheless $\theta$ and $\theta_{f}$ are still analogous to $D q^{2}$.

Turning first to the slow mode, $\theta$ shows behavior consistent with diffusive probe motion, especially for large probes. In particular, for all probes $\theta=a q^{2}$ and $\theta \rightarrow 0$ as $q^{2} \rightarrow 0$, these behaviors being signatures for a diffusive process. Furthermore, $\beta=1$ for the large probes, so for large (but not small) probes $\theta$ is a true decay rate. For simple Stokes-Einstein diffusion, $D$, the solution viscosity $\eta$, and the probe diameter $d$ are correlated via $D \sim(d \eta)^{-1}$. As seen in Figure 13, for large probes, $\theta \eta$ is constant to within a factor of two or three, so that $D \sim(d \eta)^{-1}$, as expected for Stokes-Einsteinian diffusion. However, for small probes, $\theta \eta$ increases by up to 400 fold with increasing $c . \theta$ depends on concentration via a stretched exponential $\exp \left(-\alpha c^{\nu}\right)$. For small probes, $\nu \approx 1$; for large probes $\nu$ declines substantially with increasing $d$.

The scaling pre-factor $\alpha$ from the concentration dependence of $\theta$ (Eq. 12) depends on $d$, as seen in Figure 14. If the slow mode was diffusive, $\alpha$ would be independent of $d$ and would coincide with the viscosity pre-factor $\alpha_{\nu}$. For very large spheres, values of $\alpha$ are close to 1 , but there is still some deviation of $\theta$ from Stokes-Einsteinian behavior. Because the precision of the $\alpha$ determination is not extremely high, $\alpha$ would not be expected in real data to be exactly equal to $\alpha_{\nu}$.

$\theta_{f}$ lacks the properties expected of diffusive rate constants. $\theta_{f}$ depends on scattering vector as $a q^{2}+b$. In some cases, the intercept $b \neq 0$; in other cases, the dependence of $\theta_{f}$ on $q^{2}$ is only piecewise, so that $\theta_{f}$ has one linear dependence on $q^{2}$ at small $q^{2}$, and a different linear dependence at large $q^{2}$. $\theta_{f}$ does depend on probe $d$, but not in a simple way: $\theta_{f}$ is nearly independent of $d$ for small $(<40 \mathrm{~nm})$ and for large $(>100 \mathrm{~nm})$ probes, but changes by roughly 10 fold between these size regimes. Finally, in contrast to $\theta, \theta_{f}$ in general increases weakly with $c ; \theta_{f}$ therefore does not track $\eta^{-1}$.

The fractional amplitude $A_{f}$ of the fast mode depends strongly on both polymer concentration and sphere size. In the limit of low $c$ and $90^{\circ}$ scattering, $A_{f}$ is very close to zero. $A_{f}$ increases with increasing $c$, to $\approx 0.3$ for small spheres, and to $\approx 0.6$ for intermediate size spheres. For large spheres, $A_{f}$ reaches a peak value of 0.6 for $c \approx 4 \mathrm{~g} / \mathrm{L}$; at larger $c, A_{f}$ decreases again. The $q^{2}$ dependence of $A_{f}$ is not strongly dependent on $c$, but depends on probe $d$. For small spheres, $A_{f}$ decreases sharply with increasing $q^{2}\left(A_{f} \rightarrow 1\right.$ as $\left.q \rightarrow 0\right)$, while for intermediate and large spheres $A_{f}$ is nearly independent of $q^{2}$ or increases modestly with increasing $q^{2}$.

We propose that the slow and fast modes may be interpreted as probe diffusion through a non-simple medium, and as probe motion coupled to internal chain modes, respectively. We further propose that the probe-polymer coupling in each modes is qualitatively different for small and large spheres. The fundamental length separating "small", and "large", probes is the size of a polymer chain.

We first summarize evidence that the slow mode reflects diffusion. Note in particular the diffusive $q^{2}$ dependence of $\theta$, and for large probes the simple-exponential relaxation $\exp \left(-\theta t^{1}\right)$ with $\theta \sim(d \eta)^{-1}$. For smaller probes, $\theta$ no longer depends simply on $\eta$ or $d$. However, the slow mode of smaller probes appears to represent the continuous extension to smaller $d$ of the slow mode of larger probes; note in particular that $\theta \sim a q^{2}$ and at larger $q, A_{f} \rightarrow 0$ as $c \rightarrow 0$ for all probe sizes.

Russo et al. ${ }^{12}$ have studied probe diffusion using probes of different size, most of their probes being smaller than ours. Russo et al. found that $D \eta$ increased with increasing $c$, but that $D \eta$ was nearly constant (i.e., was closest to StokesEinsteinian behavior) for the largest probes that they examined. We observe a fast as well as a slow relaxation, (perhaps because our experimental method is more sensitive to short times), but our results on $\theta$ extend their findings to larger probes. As $d$ increases from Russo et al.'s largest probes to our larger probes, $D \eta$ reaches a $c$-independent constant, precisely as Russo et al. ${ }^{12}$ proposed on the basis of assumed diffusive behavior.

Our argument that the fast mode reflects coupling to chain modes is indirect. The fast mode has properties expected, according to the Ngai coupling model, ${ }^{8,9}$ for chain internal motions. In terms of Ngai's model, here the individual diffusing units are polymer chains or segments, and the interactions between individual units are chain-chain or segment-segment interactions. These interactions increase in strength with increasing $c$. The Ngai model correctly indicates that the relaxation function of the fast mode is a stretched exponential in time, and correctly predicts that the coupling parameter $\beta_{f}$ declines with increasing $c$. Furthermore, the decay constant $\theta_{f}$ increases weakly with $c$, but only depends substantially on $d$ within a small-to-large probe transition regime. A mode whose dynamics were primarily determined by the motion of chains, and in which the probes were so-to-speak passive bystanders awaiting motion by the chains, could readily have these properties. $\theta_{f}$ would be enhanced by repulsive excluded-volume contacts between chain segments, so it could increase with increasing $c$. However, a $\theta_{f}$ that was determined by chain-chain motions could be substantially independent of $d$, the small-to-large probe transition of $\theta_{f}$ appearing because all small probes sample the same local chain modes, while large probes also see whole-chain modes. The $c$-dependence of $A_{f}$ is also consistent with the polymer-coupling interpretation of $\theta$, because internal chain motions ought to be more effectively coupled when there are more chains.

A significant difficulty with interpreting the fast mode as arising from internal chain motions is the lack of a mechanism to couple chain motion-as opposed to the presence of nearby chains - to probe motion or the scattering spectrum. At the simple coupled-diffusion level, one may write for two interacting species $\mathrm{A}$ and $\mathrm{B}$

$$
\begin{aligned}
& \frac{d c_{A}}{d t}=D_{A A} \nabla^{2} c_{A}+D_{A B} \nabla^{2} c_{B} \\
& \frac{d c_{B}}{d t}=D_{B A} \nabla^{2} c_{A}+D_{B B} \nabla^{2} c_{B} .
\end{aligned}
$$

Here $c_{A}$ and $c_{B}$ are the position- and time-dependent concentrations of the two species, while $D_{A A}$ and $D_{B B}$ are their 
diffusion coefficients; $D_{A B}$ and $D_{B A}$ are cross-diffusion coefficients. As first shown by one of us ${ }^{26}$ the light scattering spectrum of such a system in general has two modes, the relaxation rate of each mode being determined by all four $D_{i j}$. Similar results for special cases have since been reported, e.g., by Jones. ${ }^{27}$

Even if only one species scatters any light, both modes are visible in the scattering spectrum. ${ }^{26}$ However, if the scattering species is also dilute, then as first shown in Refs. 27,28 one spectral mode disappears. The spectrum contains a single mode reflecting single-particle diffusion of the scatterers through an unseen background. Our scatterers are highly dilute, but two modes still appear, a result that does not follow from eqs. 14 and 15. An explanation for our observations is that polymer motions couple to probe diffusion via a frequency dependent effective viscosity as discussed by Wang. ${ }^{29}$ Wang, however, emphasizes that the mechanism he envisaged is not effective if the volume of mixing of the macro-components is small, as appears likely in our system.

Slow and fast modes may also be described qualitatively within the framework of the Mori-Zwanzig ${ }^{30}$ memoryfunction formalism, in which the relaxation of a variable $A$ may be written

$$
\frac{d A}{d t}=i \Omega A-\int_{0}^{s} d s K(t-s) A(s)+f(s) .
$$

We are in an equilibrium system with time-reversal symmetry, so the frequency function $\Omega$ always vanishes. Here $K$ is the memory function for $A$, and $f$ is the random force. $K$ is also the time correlation function of $f$. In a simple diffusive process, $K$ is a delta function in time and $\langle A(t) A(0)\rangle$ decays exponentially in $t$.

For all probes, $g^{(1)}(t)$ is the sum of a slow and a fast mode. For large probes, the slow mode is decaying exponentially for times as small as a few $\mu$ sec. An exponential decay corresponds to a delta-function memory; we are obliged to infer for large probes that the slow mode memory function decays near-instantaneously ( $<\mathrm{a}$ few $\mu \mathrm{sec}$ ) to zero. In contrast, for large probes the fast mode has a non-exponential decay, so the fast-mode memory function must persist over the time scales on which we make observations. Observe the sharp contrast between the modes and their memory functions; for large probes and large $c$ the slow mode persists to 10 fold longer times than the fast mode, but the slow-mode memory function is conclusively shown by our data to have a much shorter correlation time than the fast-mode memory function has.

For small probes, both modes have stretched-exponential decays, with $\beta>\beta_{f}$ in each system. The slow decay is closer to a pure-exponential than is the fast decay. We therefore infer that, for small as well as large probes, $K(t-s)$ for the slow decay is shorter-lived than $K(t-s)$ for the fast decay.

While the slow mode of small spheres appears to be the continuous extension to smaller $d$ of the slow mode of large probes, there are several prominent differences between the small and large probe behaviors. For large spheres, $\theta$ has a strong stretched-exponential dependence on $c$, while $\beta=1$; in contrast, for small spheres, $\theta$ has almost no $c$-dependence, while $\beta$ falls from 1.0 to 0.7 with increasing $c$. Furthermore, $\theta \eta$ for large probes is substantially independent of $c$, while for small probes $\theta \eta$ increases sharply with $c$.

There are also differences between the fast modes of small and large probes. For small spheres $\theta_{f}$ is an order of magnitude larger than $\theta_{f}$ of large spheres. Furthermore, $\theta_{f}$ has a far stronger $q$ dependence for small than for large spheres, and perhaps is $\approx 0$ for small spheres at low $q^{2}$. While $A_{f}$ of larger spheres increases only weakly with $q, A_{f}$ of small spheres has a very strong $q$ dependence. For small spheres, the fast mode is clearly dominant for motion over large distances (small $q$ ), and is less important over small distances (large $q$ ) ; indeed, our data is consistent with $A_{f}$ $\rightarrow 1$ as $q \rightarrow 0$. For small spheres, the dominance of the fast mode at large distances ( small $q$ ) increases with rising $c$, while at small distances (large $q$ ) the influence of the fast decay is practically the same at all $c$.

Finally, the average size of our HPC chains follows from Yang and Jamieson, ${ }^{18}$ who used static and quasi-elastic light scattering to determine $R_{g}$ and $\left\langle R_{h}^{-1}\right\rangle^{-1}$ for HPC samples having $110 \leqslant M_{w} \leqslant 850 \mathrm{kDa}$. Extrapolating their results to our $M_{w}$, we infer that our $1 \mathrm{MDa}$ polymer had $2 R_{g} \approx 210$ $\mathrm{nm}$ and $2\left\langle R_{h}^{-1}\right\rangle^{-1} \approx 110 \mathrm{~nm}$. The transition from small- to large-sphere behavior in our data clearly does not begin for probe diameters $d$ below $40 \mathrm{~nm}$, and has substantially completed at probe diameters ca. $100 \mathrm{~nm}$. Our small-to-large transition in probe phenomenology begins as $d$ approaches $R_{h}$ of the chains, consistent with the fundamental importance of hydrodynamic interactions in probe diffusion. The transition process is substantially complete for $d>R_{g}$. The fundamental length scale for these systems is thus seen to be established by the size of an entire polymer chain.

\section{ACKNOWLEDGMENTS}

The partial support of this work by the National Science Foundation under Grant No. DMR94-23702 is gratefully acknowledged.

${ }^{1}$ P.-G. de Gennes, J. Chem. Phys. 55, 572 (1971). P.-G. de Gennes, Scaling Concepts in Polymer Physics (Cornell University Press, Ithaca, 1979).

${ }^{2}$ M. Doi and S. F. Edwards, The Theory of Polymer Dynamics (Oxford University Press, Oxford, 1986).

${ }^{3}$ G. D. J. Phillies, J. Phys. Chem. 93, 5029 (1989).

${ }^{4}$ J. Skolnick and A. Kolinski, Adv. Chem. Phys. 78, 223 (1990).

${ }^{5}$ G. D. J. Phillies, J. Phys. Chem. 96, 10061 (1992).

${ }^{6}$ G. D. J. Phillies and C. Quinlan, Macromolecules 28, 160 (1995).

${ }^{7}$ G. D. J. Phillies, C. Richardson, C. A. Quinlan, and S.-Z. Ren, Macromolecules 26, 6849 (1993).

${ }^{8}$ K. L. Ngai, A. K. Rajagopal, and S. Teiler, J. Chem. Phys. 88, 5086 (1988).

${ }^{9}$ K. L. Ngai and R. W. Rendell, J. Mol. Liquids 56, 199 (1993).

${ }^{10}$ K. L. Ngai, R. W. Rendell, A. K. Rajagopal, and S. Teiler, Ann. (N.Y.) Acad. Sci. 484, 150 (1985).

${ }^{11}$ B. Nystrom, J. Roots, A. Carlsson, and B. Lindman, Polymer 33, 2875 (1992)

${ }^{12}$ Z. Bu and P. S. Russo, Macromolecules 27, 1187 (1994).

${ }^{13}$ G. D. J. Phillies and D. Clomenil, Macromolecules 26, 167 (1993).

${ }^{14}$ R. Furukawa, J. L. Arauz-Lara, and B. R. Ware, Macromolecules 24, 599 (1991).

${ }^{15}$ A. K. Rizos, T. Jian, and K. L. Ngai, Macromolecules 28, 517 (1995); K. L. Ngai, Y.-N. Wang, and L. B. Magalas, J. Alloys Compd. 211/212, 327 (1994).

${ }^{16}$ B. Nystrom, H. Walderhaug, and F. K. Hansen, J. Phys. Chem. 97, 7743 (1993).

${ }^{17}$ W. Brown and R. Rymden, Macromolecules 19, 2942 (1986). 
${ }^{18}$ T. Yang and A. M. Jamieson, J. Colloid Interface Sci. 126, 220 (1988).

${ }^{19}$ M. B. Mustafa and P. S. Russo, J. Colloid Interface Sci. 129, 240 (1989).

${ }^{20}$ M. B. Mustafa, D. L. Tipton, M. D. Barkley, P. S. Russo, and F. D. Blum, Macromolecules 26, 370 (1993).

${ }^{21}$ P. S. Russo, M. Mustafa, T. Cao, and L. K. Stephens, J. Colloid Interface Sci. 122, 120 (1988).

${ }^{22}$ G. D. J. Phillies and M. Lacroix, J. Phys. Chem. 101, 39 (1997).

${ }^{23}$ G. D. J. Phillies, Rev. Sci. Instrum. 67, 3423 (1996).

${ }^{24}$ D. E. Koppel, J. Chem. Phys. 57, 4814 (1972).

${ }^{25}$ J. H. Noggle, Physical Chemistry on a Macrocomputer (Little Brown,
Toronto, 1985).

${ }^{26}$ G. D. J. Phillies, J. Chem. Phys. 60, 976 (1974).

${ }^{27}$ R. B. Jones, Physica (Utrecht) 97A, 113 (1979).

${ }^{28}$ G. D. J. Phillies, Biopolymers 14, 499, (1975); K. Ullmann, G. S. Ullmann, and G. D. J. Phillies, J. Colloid Interface Sci. 105, 315 (1985).

${ }^{29}$ C. H. Wang, in Dynamic Light Scattering, edited by W. Brown (Clarendon Press, Oxford, UK, 1993), Chap. 5.

${ }^{30}$ H. Mori, Prog. Theor. Phys. 33, 423 (1965); R. Zwanzig, Phys. Rev. 124, 983 (1961). 
Journal of Chemical Physics is copyrighted by AIP Publishing LLC (AIP). Reuse of AIP content is subject to the terms at: http://scitation.aip.org/termsconditions. For more information, see http://publishing.aip.org/authors/rights-and-permissions. 